https://helda.helsinki.fi

\title{
Effect of driving experience on anticipatory look-ahead fixations in real curve driving
}

\section{Lehtonen, Esko}

2014-09

Lehtonen , E , Lappi , O , Koirikivi , I \& Summala , H 2014 , ' Effect of driving experience on anticipatory look-ahead fixations in real curve driving ' , Accident Analysis and Prevention , vol. 70 , pp. 195-208 . https://doi.org/10.1016/j.aap.2014.04.002

http://hdl.handle.net/10138/136215

https://doi.org/10.1016/j.aap.2014.04.002

acceptedVersion

Downloaded from Helda, University of Helsinki institutional repository.

This is an electronic reprint of the original article.

This reprint may differ from the original in pagination and typographic detail.

Please cite the original version. 


\title{
Effect of driving experience on anticipatory look-ahead fixations in real curve driving
}

\author{
Esko Lehtonen ${ }^{\mathrm{a}, \mathrm{b}}$ \\ Otto Lappi ${ }^{\text {b, a }}$ \\ livo Koirikivi ${ }^{\text {a }}$ \\ Heikki Summala ${ }^{a}$ \\ ${ }^{a}$ Traffic Research Unit, Institute of Behavioural Sciences, University of Helsinki, Finland \\ ${ }^{\mathrm{b}}$ Cognitive Science, Institute of Behavioural Sciences, University of Helsinki, Finland
}

Corrresponding author:

Esko Lehtonen

esko.lehtonen@helsinki.fi

+358 919129421 (phone)

Traffic Research Unit

Siltavuorenpenger $1 \mathrm{~A}$

FIN-00014 University of Helsinki

Finland

NOTICE: This is the author's version of a work that was accepted for publication in Accident Analysis and Prevention.. Changes resulting from the publishing process, such as peer review, editing, corrections, structural formatting, and other quality control mechanisms may not be reflected in this document. Changes may have been made to this work since it was submitted for publication. A definitive version was subsequently published in Accident Analysis and Prevention, 70 , September 2014. http://dx.doi.org/10.1016/j.aap.2014.04.002 


\begin{abstract}
Anticipatory skills are a potential factor for novice drivers' curve accidents. Behavioural data show that steering and speed regulation are affected by forward planning of the trajectory. When approaching a curve, the relevant visual information for online steering control and for planning is located at different eccentricities, creating a need to disengage the gaze from the guidance of steering to anticipatory look-ahead fixations over curves. With experience, peripheral vision can be increasingly used in the visual guidance of steering. This could leave experienced drivers more gaze time to invest on look-ahead fixations over curves, facilitating the trajectory planning.

Eighteen drivers (nine novices, nine experienced) drove an instrumented vehicle on a rural road four times in both directions. Their eye movements were analyzed in six curves. The trajectory of the car was modelled and divided to approach, entry and exit phases.

Experienced drivers spent less time on the road-ahead and more time on the look-ahead fixations over the curves. Look-ahead fixations were also more common in the approach than in the entry phase of the curve. The results suggest that with experience drivers allocate greater part of their visual attention to trajectory planning.
\end{abstract}

\title{
Keywords
}

steering, anticipation, visual attention, driving experience, hierarchical control, real driving 


\section{Introduction}

Young drivers have a higher risk of accidents in curves (Clarke et al., 2006; Adbel-Aty and Radwan, 2000). For young males, curve accidents are often loss of control accidents which involve excessive speed (Clarke et al., 2006; Laapotti and Keskinen, 1998). While excessive speed is often chosen deliberately, another contributing factor for curve accidents could be novice drivers' poorer trajectory planning skills (choice of path and speed). In the current study, we study the effect of driving experience on look-ahead fixations over curves. Look-ahead fixations can be interpreted to serve trajectory planning by providing information on the future roadway and oncoming cars (Lehtonen et al., 2012, 2013).

\subsection{Visual control of steering}

In visually guided locomotion like driving, gaze is mostly toward the intended direction of locomotion, leading direction changes with a small preview time (e.g. Land and Lee, 1994; Imai et al., 2001; Bernardin et al., 2012; Vansteenkiste et al., 2013). In car driving, the visual preview in curves is typically approximately $2 \mathrm{~s}$ (Mars, 2008). In many steering models, this "looking where you are going" behaviour is interpreted as fixating on a steering point, e.g. the tangent point or some point on the future path (Salvucci and Gray, 2004; Wilkie et al. 2008; Boer, 1996; Land and Lee, 1994).

There are two main types of mechanisms proposed for the use of a steering point (for review, see Wann and Land, 2000; Steen et al., 2011; Lappi et al., 2013). The first type of mechanism proposes that the direction of the steering point or its change is used to adjust the steering (e.g. Land and Lee, 1994), as from the steering point it is possible to calculate the instantaneous curvature of the road. The second type of steering mechanism uses the retinal flow and active gaze to control the steering so that the driver will travel along a constantly curved path from the current location to the fixated steering point location (Wann and Swapp, 2000; Boer, 1996).

Because fixations towards the road ahead with a small anticipatory lead time are thought to guide steering, we call these guiding fixations. With this naming, we intentionally relate them to the guiding fixations reported in many well-learned visually guided manipulation tasks. In these tasks, gaze is mostly directed to objects or locations relevant for guidance of the current action (e.g. Land et al. 1999; Pelz and Canosa 2001; Hayhoe et al. 2003). This guidance of action is done typically in a just-in-time fashion (Ballard et al. 1995), which means that gaze is directed according to the immediate needs of the current task phase, leading motor action with a small time margin $(<2 \mathrm{~s})$.

There is empirical and modelling support for usage of multiple steering points at different preview distances. In two-level steering models (Donges, 1978, Land and Horwood, 1995; Salvucci and Gray, 2004; Frissen \& Mars, 2013; for a critical view, see Cloete and Wallis, 2011) the steering mechanisms outlined earlier correspond to the guidance level of steering, which uses steering points in the far zone. In addition to the guidance level, there is the stabilizing level of steering, which utilizes steering points close to the car from the near zone. The stabilizing level of steering helps to maintain lane-position more accurately, and the guidance level is important for smoothness of steering (Land and Horwood 1995; Salvucci and Gray 2004). With experience, lanekeeping becomes possible with peripheral vision (Mourant and Rockwell, 1972; Summala, 1998; Summala et al., 1996) and thus most guiding fixations are directed toward the steering point in the far zone. 
The steering models above are online control models, where current visual information is translated into the immediate steering response. However, online steering control is only part of the hierarchical control of the driving task (e.g. Michon, 1985; Summala, 1997). In the hierarchical control of actions, the higher levels set goals which are accomplished by a sequence of actions controlled by the lower levels (e.g. Cooper and Shallice, 2000; Grafton and Hamilton 2007; Land 2009).

\subsection{Trajectory planning}

In curve driving, trajectory planning can be thought as a control level superior to the guidance level of steering. Here, we used the term trajectory as a compound of path ${ }^{1}$ and speed, because the two are inherently linked to each other. For example, cutting a corner allows higher speed. In trajectory planning, a driver utilizes visual information of the curve and of other road users to perceive an affordance for locomotion. The roadway and other road users place both static and dynamic constraints for locomotion (Gibson and Crooks, 1938; Fajen and Warren, 2003: Summala, 2007), and a trajectory plan can be thought as a solution which can satisfy these constraints. Of course, a trajectory plan is also affected by the performance of the vehicle and skills and motives of the driver.

As the situation unfolds, the trajectory plan can be updated - for example when an oncoming car emerges - if necessary and sufficient attentional capacity and time is available. In other words, trajectory planning can be thought also as a process for anticipatory maintenance of safety margins or a safety zone (Gibson and Crooks, 1938; Summala, 2007). For example, by choosing a trajectory with a low enough speed drivers can increase their time-to-line crossing (Godthelp et al., 1984).

One of the functions of a trajectory plan is anticipatory adjustment of speed before entering a curve (Hassan and Sarhan, 2012; Cruzado and Donnell, 2010; Charlton, 2007; Shinar et al., 1980). Another is anticipation of other road users, especially oncoming cars (Muttart et al., 2013; Lehtonen et al., 2012), which constrain the choice of path and speed.

As superior to the guidance and stabilization level of steering (Donges, 1978; Salvucci and Gray, 2004), trajectory planning is able to preprogram motor actions and execute them even in the absence of continuous visual feedback. Cavallo et al. (1988) demonstrated that drivers are able to time the steering wheel rotation correctly even when the visual field was occluded $2 \mathrm{~s}$ before entering a curve. Furthermore, experienced drivers $(>100000 \mathrm{~km})$ were able to match size of the steering wheel rotation correctly under occlusion, while novices underestimated the required rotation. Without occlusion, there was no difference between learner drivers and experienced, because visual feedback was available for online control of steering to complete the trajectory plan.

Trajectory planning needs visual information from the roadway and other road users. Anticipatory eye movements toward the direction of the curve in driving have been often reported (Cohen and Studach, 1977; Shinar et al., 1977; Land and Horwood, 1996; Lehtonen et al., 2012, 2013; Mars and Navarro, 2012; Muttart et al. 2013; cf. Marigold and Patla, 2007 for trajectory planning in walking). However, the definition of an anticipatory eye movement toward a curve has varied, and is problematic, because often the same fixations can support both guidance and trajectory planning.

\footnotetext{
${ }^{1}$ For variation in choice of path in natural driving, see Spacek (2005).
} 
While approaching a curve on a straight road, a fixation approximately straight ahead, toward the entry of the curve, can provide information for all three levels. In this case, the near zone is at relatively low eccentricity which makes it feasible to use peripheral vision for the stabilising level (Summala, 1998; Summala et al., 1996), and a steering point in the far zone can be monitored. Also, the entry point of the curve is in the same direction, which makes it possible to anticipate the right moment of the curve entry. However, the rest of the curve which carries essential visual information from the horizontal and vertical curvature is not visible foveally when looking straight ahead.

\subsection{Look-ahead fixations over curves}

In order to acquire accurate foveal information from the rest of the curve, drivers need to make an eccentric fixation toward the road further up, disengaging the gaze from the visual guidance of online control of steering. These fixations have been called look-ahead fixations (Lehtonen et al., 2013; Mars and Navarro, 2012), relating these fixations to the look-ahead fixations reported in visually guided sequential manipulations task, where look-ahead fixations are often done toward the objects or locations relevant to a future task phase, but gaze is quickly returned back to the guidance of the current task phase (e.g. Pelzand Canosa, 2001; Mennie et al., 2007).

In curve driving, look-ahead fixations could help to construct and update the trajectory plan. In particular, look-ahead fixations are unlikely to be driven by the visual requirements of guidance level of steering. The guidance level models posit that the steering is adjusted relative to the steering point with a 1-2 s preview time so that a vehicle will travel along a constantly curved path, maintaining the lane position (e.g. Land and Lee, 1994) or reaching the steering point (Wann and Swapp, 2000) depending on the model. If the steering point is selected too far along the road with variable curvature, the required steering cannot be approximated with constant curvature. This is especially prominent when approaching a curve on a straight road where a steering response must not be initiated during the straight segment when fixating a point further ahead in the curve. Similarly, this also applies when fixating beyond the curve during steering within a curve.

Because guiding fixations towards the far zone may also carry anticipatory information for trajectory planning, e.g. when approaching an entry of the curve on a straight segment, it is not always possible to completely distinguish the online control of steering and the trajectory planning at the level of the eye movements. However, it is possible to use eccentric look-ahead fixations over curves to quantify how much the drivers invest their gaze time solely on trajectory planning, and not on online control of steering. Dwell time on eccentric look-ahead fixations over curves could therefore be used as an index of trajectory planning.

As it is not entirely clear when a fixation is functionally a guiding or a look-ahead fixation, we will call all the fixations toward the road-ahead road ahead fixations. We will use the term look-ahead fixation for eccentric look-ahead fixations over curves.

\subsection{Aims of the study}

In this on-road study, we investigate look-ahead fixations when approaching and negotiating curves with an open view over the curve on a rural road. We use a model of the future trajectory to identify both the fixations toward the road-ahead and the eccentric look-ahead fixations over curves. 
We expect that experienced drivers would spend more time on look-ahead fixations over curves. Experienced drivers, in general, anticipate traffic situations better than novices (Jackson et al., 2009; Underwood, 2007). Experienced drivers are also better able to use peripheral vision for steering (Summala et al., 1996), leaving them more time to disengage their gaze from the guiding fixations. Look-ahead fixations should also be more common in the approach phase compared to the entry phase, because visual demand of steering is higher within curves (Tsimhoni et al., 2001).

Some previous studies have already linked driving experience to anticipatory scanning in curves. Cohen and Studach (1977) found that experienced drivers make larger saccades than novices in left, but not in right curves. However, they studied only curves with limited visibility. In right-side drive, the sight distance in left curves is longer than in right curves. This suggests that experienced drivers could make larger anticipatory eye movement when there is an open view over the curve. More recently, Muttart et al. (2013) studied hazard anticipation in curves in a simulator and found that experienced drivers glanced more often to "the far extent" of the curve in curves to left. Their result also suggested that this visual anticipation was linked to anticipatory speed reduction and decreased crashes among experienced drivers.

\section{Methods}

\subsection{Participants}

Nineteen drivers participated in the experiment. All the drivers held valid driver's licenses and had normal uncorrected vision or vision corrected with contact lenses. One novice driver's data was left out of the analysis, because the driver could not comfortably operate a manual transmission. The other eighteen drivers were divided into two groups based on the driving experience.

Nine drivers were classified as novices ( 3 males, 6 females, age $18-33$ years, $M=24, S D=5$ ). All of the novice drivers reported less than $5000 \mathrm{~km}$ of driving experience. 9 drivers were classified as experienced drivers ( 7 males, 2 females, age $23-30, M=26, S D=2$ ). Of the experienced drivers, one reported driving experience between 20000 and $30000 \mathrm{~km}$; four reported between 30000 and 50 $000 \mathrm{~km}$; two reported between 50000 and $100000 \mathrm{~km}$; and two reported between 200000 and $500000 \mathrm{~km}$. Approximately $30000 \mathrm{~km}$ of driving experience was decided as enough for being classified as an experienced driver, because after $30000 \mathrm{~km}$, drivers are able to use their peripheral vision effectively in maintenance of lane-position (Summala et al., 1996).

All the participants gave informed consent for participation of the study. Research settings were approved by the local ethical committee.

\subsection{Equipment}

The instrumented car was a Toyota Corolla compact sedan with a manual transmission (model year 2007). The car had a steering wheel on the left, as Finland has right side traffic. The car was equipped with a two-camera Smart Eye Pro 5.5 eye tracker (Smart Eye AB, Gothenburg, Sweden, www.smarteye.se) operating at $60 \mathrm{~Hz}$, a forward-looking video camera and a GPS receiver. Yaw rate was recorded from the vehicle CAN bus, with a Highspeed ISO 11898 can reader operating 100 $\mathrm{Hz}$. Packet contents were reverse engineered: yaw rate packets had an accuracy of $0.25 \% \mathrm{~s}$ with an update rate of at least $100 \mathrm{~Hz}$. 


\subsection{Procedure}

Participants were read instructions before the experiment, advising them to drive as they normally would, following traffic regulations. During the experiment the participant was accompanied by two researchers, one sitting beside the driver and one behind him. One of the researchers gave instructions, if necessary, to the driver, while the other monitored data collection. Interaction between researchers and the driver was avoided during the data collection.

Participants drove an $8.1 \mathrm{~km}$ segment of rural road four times in both directions. Car-following situations were avoided by waiting until there were no other vehicles visible before entering the road.

\subsection{Curves}

Recorded data was fitted to a common road representation using GPS coordinates. As a result, each data point could be supplied with a location on the road measured from the beginning of the road. Six horizontal curves were selected for further analysis (Fig. 1). Curves were on flat terrain, and had no substantial vertical curvature. During each run, each of the six curves was first driven from east to west, and the direction of the curves was to the left. After the turn-around point, the same six curves were driven from west to east, and the direction of the curves was then to the right. There were open fields to the south of the road, and therefore all the curves afforded a good view over the curve.

The curve entry, maximum yaw rate and exit points were algorithmically detected using the yaw rate of the car, detected individually for each driver and run. In the detection algorithm, the high frequency components of the yaw rate were first removed using second-order Savitzky-Golay filter with $2 \mathrm{~s}$ window. From the smoothed signal the peaks of the signal (zero values of the derivative, with absolute value of the yaw rate larger than $3 \%$ ) were recognized. The peak with the largest absolute value was assigned as the maximum yaw rate point of the curve. The entry point was defined as the last point before the peak point where the yaw rate was less than $1 \%$ for at least $250 \mathrm{~ms}$ and its rate of change was less than $6 \% \mathrm{~s}$ (when coming from a straight segment) or where the signal crossed zero level (when coming from another curve). The curve exit point was similarly detected as the first point after the peak where the requirements were met.

The entry phase was defined as being between the entry and the maximum yaw rate points, and the exit phase between maximum yaw rate and exit points. In 4 curves a straight approach phase preceding the entry point was manually identified.

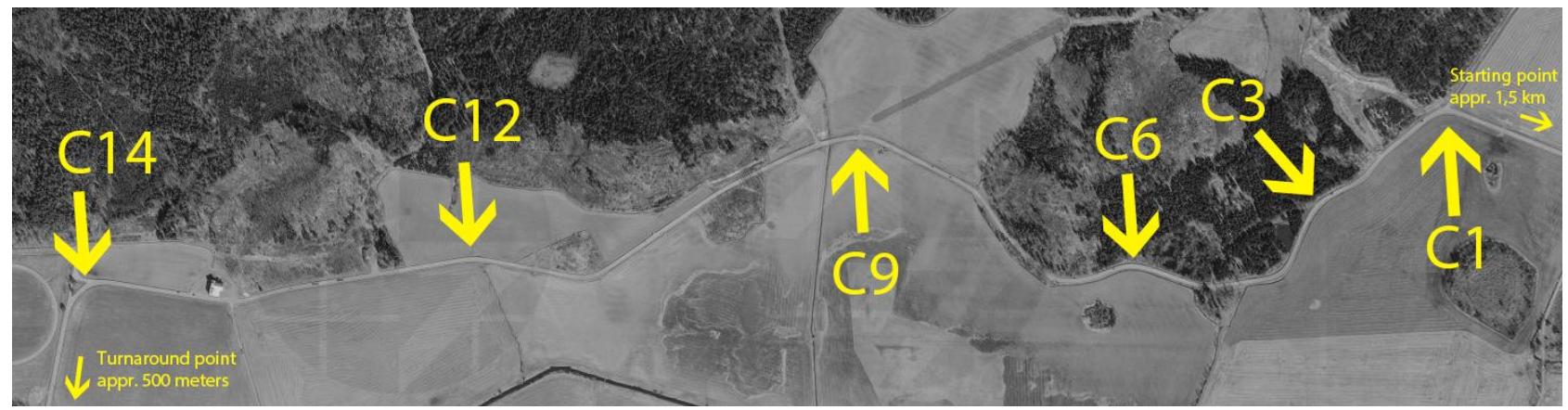

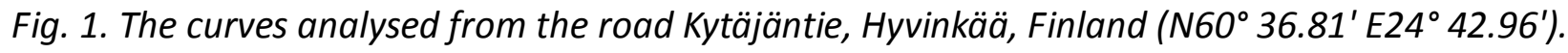




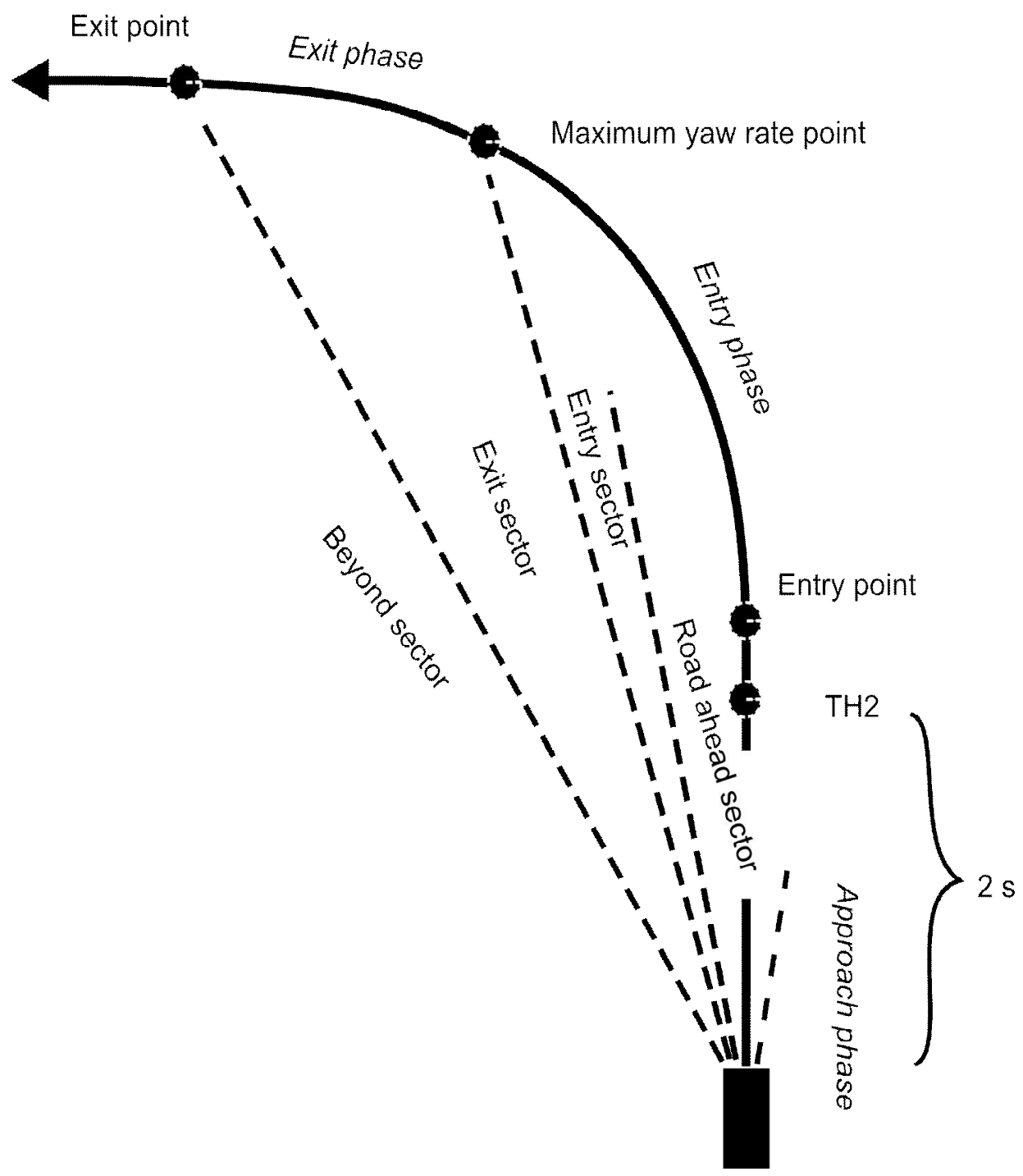

Fig. 2. Illustration of the central concepts for structuring the data. The figure shows the trajectory (line with arrow) of the vehicle (black box). Along the trajectory, there are the entry, maximum yaw rate and exit points of the curve. These points segment the trajectory to approach, entry and exit phases. The time-headway point with 2 s lead time (TH2) is also marked. From the driver's point of view, the direction of these points define sectors later used for quantification of the gaze behaviour (road ahead, entry, exit, beyond sectors)

\subsection{Eccentricity of points on the trajectory}

$2 \mathrm{D}$ approximation of the trajectory of the car along the road was calculated using speed and unsmoothed yaw rate data using the same method as in Lehtonen et al. (2013). Using the representation, we calculated the eccentricities of certain points on the trajectory at each point in time, relative to the car's heading (see Fig. 2). These points included the maximum yaw rate point, the exit point and time headway points where the car would arrive within 1, 2, 3, and $4 \mathrm{~s}$. 


\subsection{Fixation detection}

The eye tracker was calibrated using the manufacturer's calibration procedure and designated points in the car. In such a setup it is difficult to define a priori what is "straight ahead", so the origin of the gaze relative to the vehicle centreline was run-wise set to the mode of the 2D gaze distribution from straight road segments (cf. Ahlström et al., 2012). In the results we will refer to this straight ahead direction as the vehicle centreline direction. Locations of left and rear view mirror and the instrument panel were also individually determined from the 2D gaze distribution.

Fixations were detected using IV-T velocity-based detection algorithm (see Salvucci and Goldberg, 2000). The velocity threshold was set to be 0.8 times the standard deviation of intersample difference for eccentricity and 2.0 times the standard deviation for the declination. The method and criteria were chosen based on visual inspection of the detection results in order to include pursuit-like "fixations" ${ }^{2}$. Eye tracking quality deteriorated when the gaze was very eccentric, which made detection of mirror and speedometer fixation unreliable. Therefore, all the segments of data points where the values were either less than 20 degrees below the origin or more than 35 degrees eccentric from the origin were identified as single fixations.

We also calculated where the direction of the fixation crossed the future trajectory on the road, using the method described in Lehtonen et al. (2013). With this method, it was possible to estimate the crossing of the vector representing the direction of a fixation and the road ahead (in vehicle centred frame of reference) in a 2D plane. Crossing estimates were used to identify fixations which were in the direction of the curve but not directed towards the road ahead.

\subsection{Data processing}

In the design, we had 864 trials (runs through a curve, 18 participants $\times 4$ runs $\times 2$ directions $\times 6$ curves). The computers had power supply problems during two runs when driving westward. This lead to loss of 12 trials $(2 \times 6)$ of eye tracker data, and 6 trials $(1 \times 6)$ of other car data. From the remaining of 852 trials of eye tracker data 48 were excluded due to insufficient recording quality. All the curves had at least 2 trials from each participant, while most had all 4 . Finally, there were 804 (93 \%) trials available for eye tracking analyses and 858 (99\%) for car data analyses.

Most of the statistical analysis was done using mixed effect models, which allow incomplete data. Participant was modelled as a random effect, and curve as a random effect nested within a participant when a trial specific fixed continuous covariate (e.g. speed) was not used. Interactions of fixed effects were included based on log-likelihood and Bayesian Information Criteria. All the statistical analysis was done in R, using package nlme (Pinheiro and Bates, 2000) for mixed effect modelling.

\subsection{Parameterization of gaze behaviour}

The majority of the fixations are close in eccentricity to the time-headway point with $2 \mathrm{~s}$ lead time (TH2) in all curve phases (Fig. 3). From Fig. 4 we can also see that median absolute deviations (i.e.

\footnotetext{
${ }^{2}$ Based on the current data pursuit movements in relation to the movement of the vehicle have already been reported in Lappi and Lehtonen (2013).
} 
half of the fixations) are within 3-4 degrees from the TH2, which is smallest among the different references. Therefore, we use the eccentricity of the TH2 as an estimate for the eccentricity of the majority of the fixations in the curves studied. When tested, there was no significant effect of run on the median deviation from $\mathrm{TH} 2$.

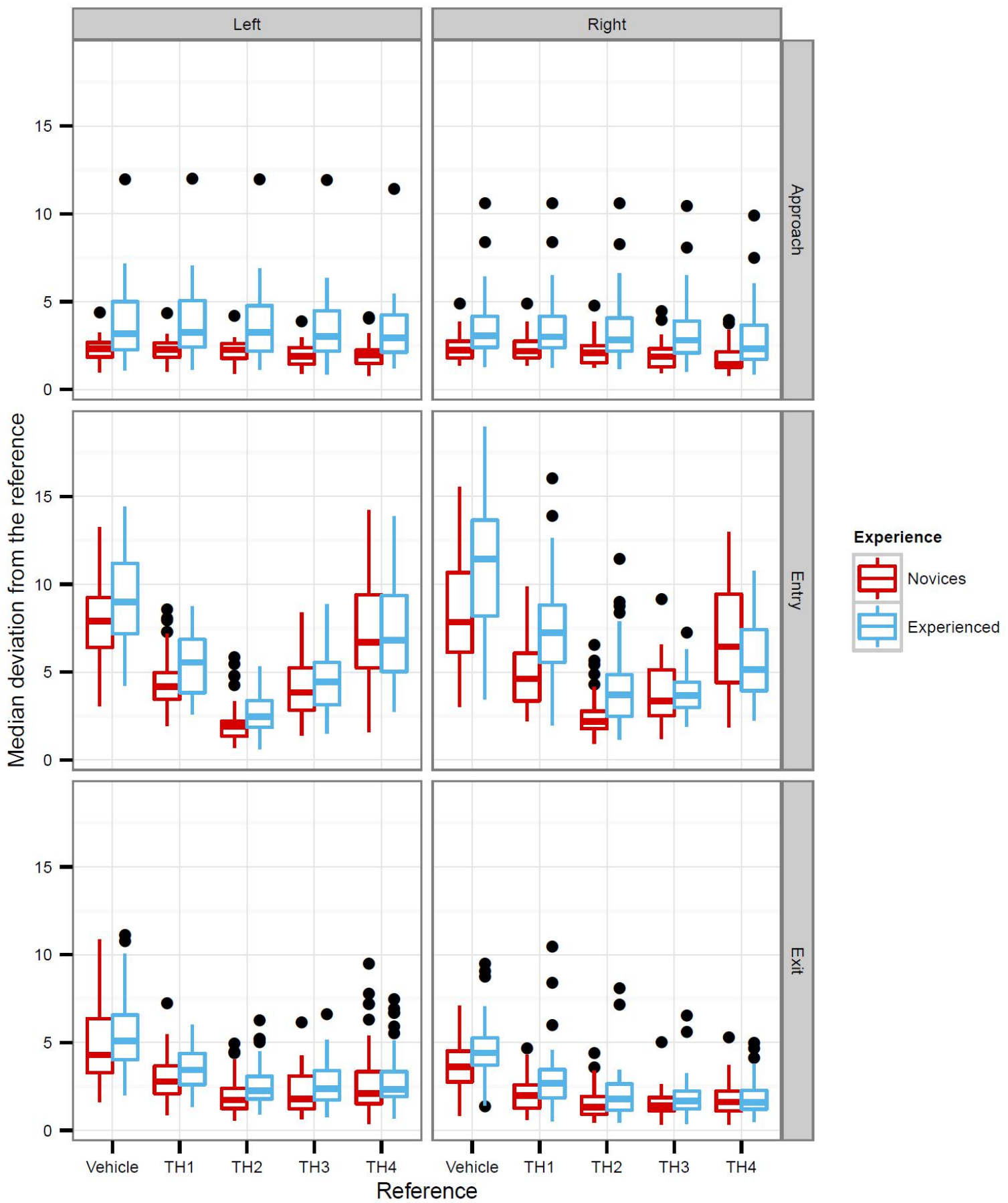

Fig. 3. Fixation eccentricities (median deviation) relative to the vehicle centreline and to different time-headway reference directions with 1, 2, 3, 4 s lead times (TH1-TH4). Median is calculated separately for each participant, curve, direction (left, right) and phase (approach, entry, exit). In the boxplots the hinges represent the first and third quartile, whiskers extend $1.5 *$ IQR of the hinge, and outliers are marked with dots.) 
Single trial data in Fig. 4a characterizes typical gaze behaviour in curves. In the approach phase, fixations are mostly straight ahead but there are some eccentric fixations over the curve.

Horizontal gaze eccentricity relative to the vehicle centreline begins to increase toward the curve in the end of the approach phase leading the vehicle rotation. Eccentricity reaches its maximum in the second half of the entry phase, after which it begins to decrease, leading the decrease in the vehicle yaw rate.

From the drivers' standpoint, the horizontal eccentricity of the maximum yaw rate point and the exit point on the future trajectory increase through the approach phase, reaching their maximum in the first half of the entry phase. After that, their eccentricities decrease. Eccentricity of the $2 \mathrm{~s}$ time headway point ( $\mathrm{TH} 2$ ) naturally anticipates the direction changes of the vehicle. In the exit phase the direction of the exit point and all of the time headway points merge to a single direction. Consequently, in the exit phase it is not necessary to make eccentric fixations in order to see the end of the curve.

For the quantification of the distribution of gaze on the road, we defined the following sectors (see also Fig. 2) as dynamical areas of interest. The road ahead sector spanned 5 degrees to both directions from the TH2 direction (from Fig. 3 shows that more than $50 \%$ of fixations being within $3-4^{\circ}$ from TH2). The entry sector consists of fixations targeting the entry phase of the curve, defined as the visual sector between the road ahead sector and the direction of the maximum yaw rate point of the curve. In practise, the road ahead sector typically covers the entry phase, making it often impossible to distinguish the road ahead fixations from those targeting the entry of the curve. The exit sector extends between the maximum yaw rate point and the exit point, excluding fixations falling to the road ahead sector. The beyond sector extends from the exit point direction to infinity, but we required that a fixation must be toward the road ahead, requiring a valid crossing point along the future trajectory. Fixations which were targeting mirrors or the instrument panel were assigned to their own areas of interest.

Finally, we defined the look-ahead fixations over the curve to be fixations which originate from the curve approach or entry phases and fall either on the curve entry, exit or beyond sectors. In other words, they are more than 5 degrees away from the $\mathrm{TH} 2$ direction and target the road further ahead.

Horizontal eccentricities of fixations classified to different sectors were plotted against the location on the road (supplementary Figs 1-12). These figures alongside the density estimates (Fig. 6) confirmed that the road-ahead sector direction (within 5 degrees from time-headway with $2 \mathrm{~s}$ lead time, $\mathrm{TH} 2$ ) captured the main body of fixations. 
(a)

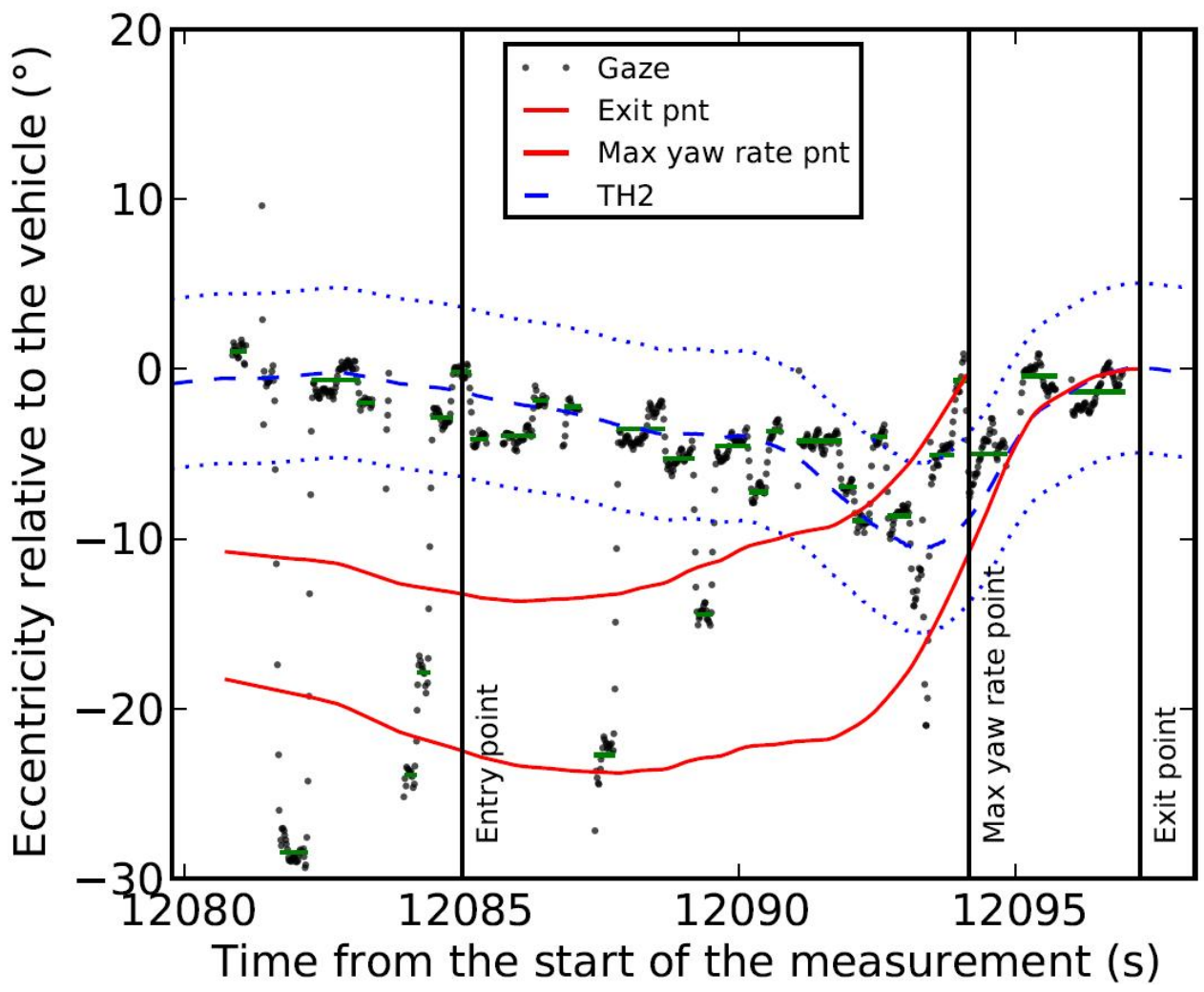

(b)

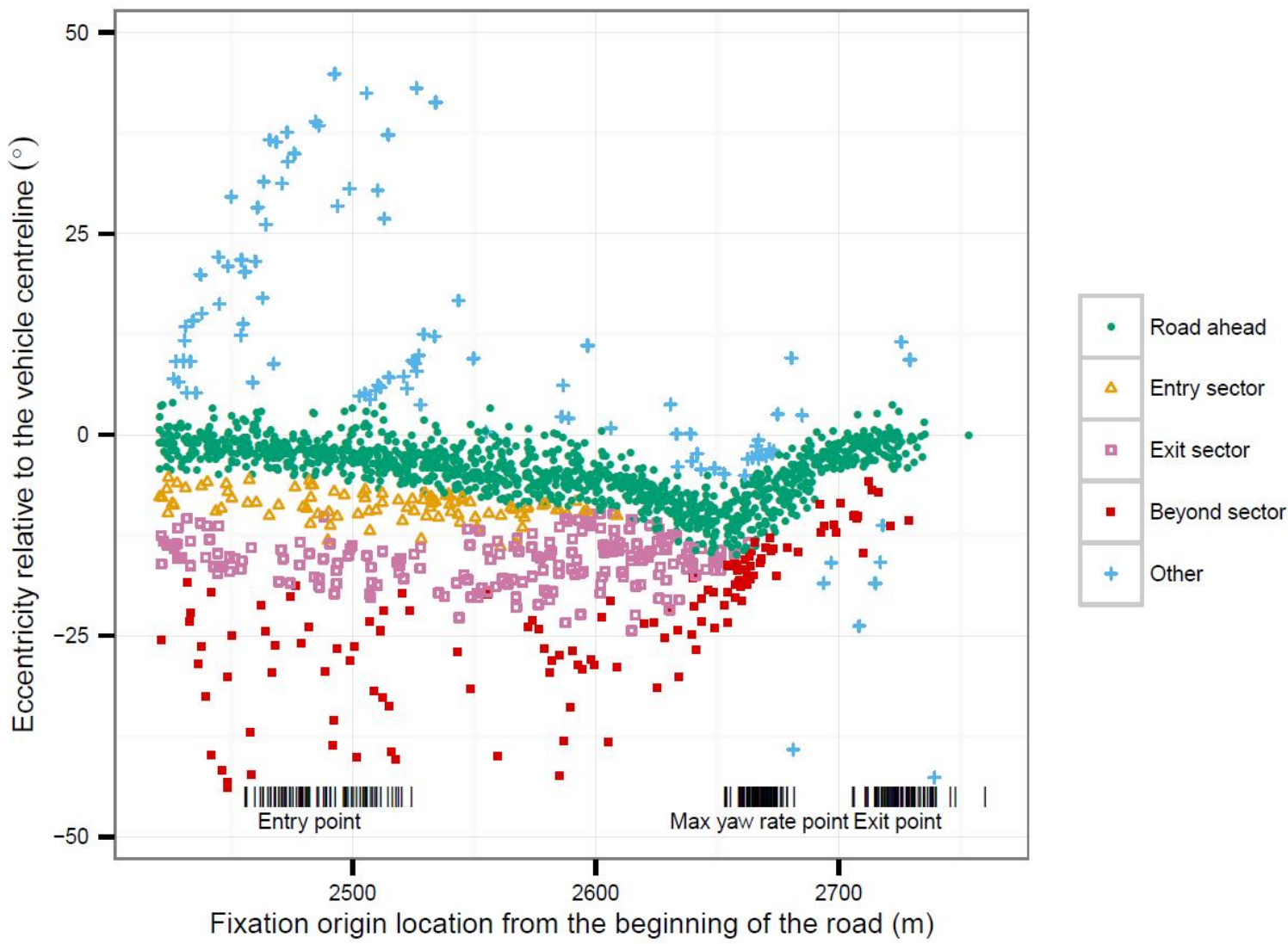


Fig. 4. a) Data from a single run though the right curve C9. Horizontal eccentricity (up: to the left) of gaze direction (black dots) relative to the directions of the maximum yaw rate and exit points (solid lines, highest: exit point, lowest: maximum yaw rate point) and to the eccentricity of the $2 \mathrm{~s}$ time headway point (TH2) with $5^{\circ}$ boundaries (dashed line). Locations of the entry, maximum yaw rate and exit points are marked with vertical lines. Detected fixations are marked over the raw gaze data with vertical lines. Time from the start of the measurement ( $x$-scale) started to run when a participant was first seated on the car. b) Origin locations of fixations and their eccentricities (up: to the left) relative to the vehicle centreline in the right curve $\mathbf{C 9}$, all fixations combined. Classification of fixations to different sectors: road ahead, entry, exit, beyond and other (scenery), is designated by shape. Fixations with different sectors may mix due to differences in individual driving lines, which affect sector boundaries. Locations of individual entry, maximum yaw rate and exit points are marked in the bottom of the figure with vertical lines.

\section{Results}

We begin the results section by describing the distribution of the fixations in different curve phases. Then we will proceed to analysis of road-ahead and look-ahead fixations. Finally, we present results related to the driving speed and steering.

\subsection{Horizontal and vertical modes}

We first wanted to investigate differences between curves, curve phases and driver groups in their guiding fixations, and evaluate how well the $2 \mathrm{~s}$ time-headway direction can be used as an estimate for direction of the road-ahead fixations. In the following results, we have excluded the fixation to the dashboard and mirrors, because we are interested in the visual control of steering.

As an estimate for the direction of the majority of guiding fixations toward the road ahead, we calculated horizontal and vertical modes of the fixation eccentricity distributions using nonparametric density estimation, separately for each driver and curve. Runs were collapsed because the density estimation is less stable with small amount of data. We also compared the horizontal modes in vehicle centred and in $2 \mathrm{~s}$ time-headway $(\mathrm{TH} 2)$ centred coordinates.

We first tested the effect of experience, curve phase and curve direction on the vehicle centred horizontal modes, treating the individual curves as random variable. Experienced drivers' modes (Fig. 5a) were more eccentric to the direction of the curve than novice drivers' $(F(1,16)=9.242$, $p=0.008$ ). The effect was most marked in the entry phase (experience $x$ phase, $F(2,336)=3.974$, $p=0.020$ ) and in right curves (experience $x$ direction, $F(2,336)=15.811, p<0.001$ ).

In a similar analysis, experienced drivers' vertical modes were higher than novice drivers', with a marginal significance $(F(1,16)=4.392, p=0.052)$. Together, these results suggest that in curves the novice drivers look closer along the curve than the experienced drivers, albeit the difference is rather small. The vertical modes were lower in the entry phase than in the approach and exit phases $(F(2,338)=117.569, p<.001)$. Also, the vertical modes were lower in left curves compared to right curves $(F(1,197)=101.023, p<.001)$, especially in entries of left curves $(F(2,338=6.334$, $\mathrm{p}=0.002$ ).

Further examination of the curvewise differences show that the speed of the curve is strongly correlated to the eccentricity of the horizontal modes in vehicle centred coordinates in curve 
entries. We tested the modes in the curve entry phase using the speed at the maximum yaw rate point as a fixed covariate, with experience and curve direction as fixed effects. Effect of speed was significant $(F(1,195)=179.376, p<0.001$, beta $=-1.19, S E=0.07)$. However, the effect of experience was also significant $(F(1,195)=11.171, p=0.004$, beta $=2.02, S E=0.84)$, indicating that the difference is not only due to the experience drivers having a higher speed (see the speed results). In entry of the right curves, the modes were more eccentric than in left curves $(F(1,195)=35.054, p<0.001)$, especially for experienced drivers $(F(1,195)=5.164, p<0.024)$.

As 2 s time-headway direction ( $\mathrm{TH} 2$ ) is expressed in time, it also controls for the differences in driving speed. We repeated the above analysis using the horizontal mode values calculated in TH2 centred coordinates. In TH2 centred coordinates (Fig. 5b), the difference between the between experienced and novices was not significant $(F(1,16)=1.816, p=0.196)$. However, the significant interaction of curve direction and experience $(F(1,196)=9.824, p=0.002)$ indicates that experienced drives look slightly further along the trajectory in right-hand curves, even when controlling for the speed.

In summary, $\mathrm{TH} 2$ appears to estimate the direction of the road-ahead fixations well, even though in the curve entries, especially to the right, the experienced drivers look slightly further relative to the $\mathrm{TH} 2$. In right-side traffic with left-hand drive, visibility to the left is restricted by the vehicle Aframe, which may underlie why difference between driver groups is smaller in left curves. 
a
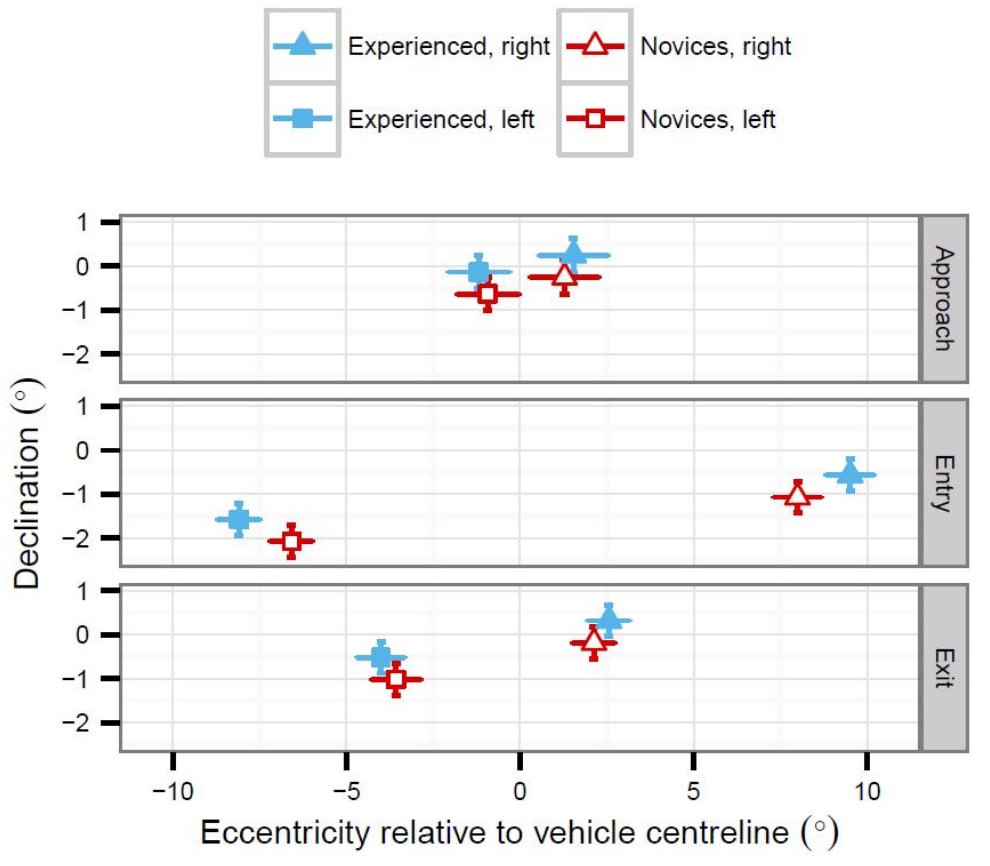

(b)
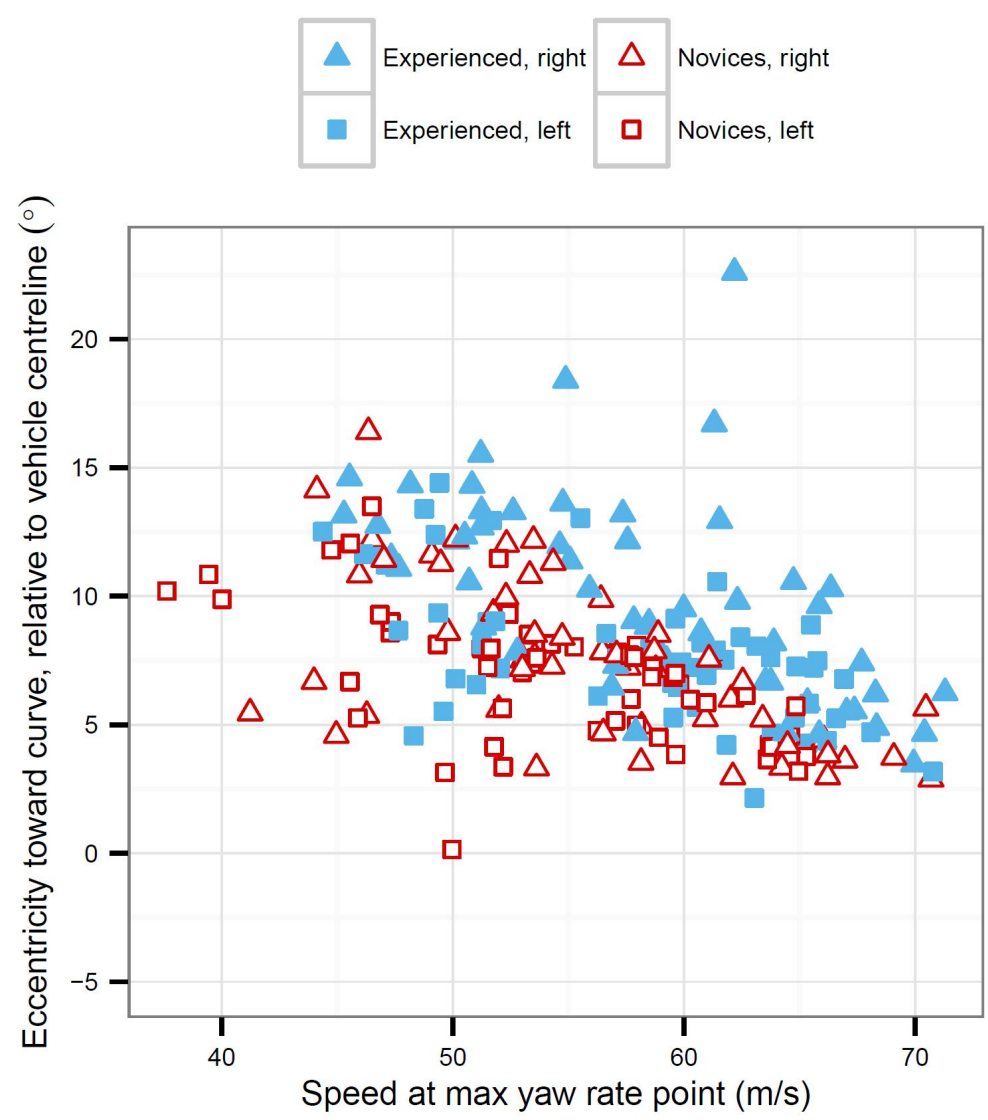
C
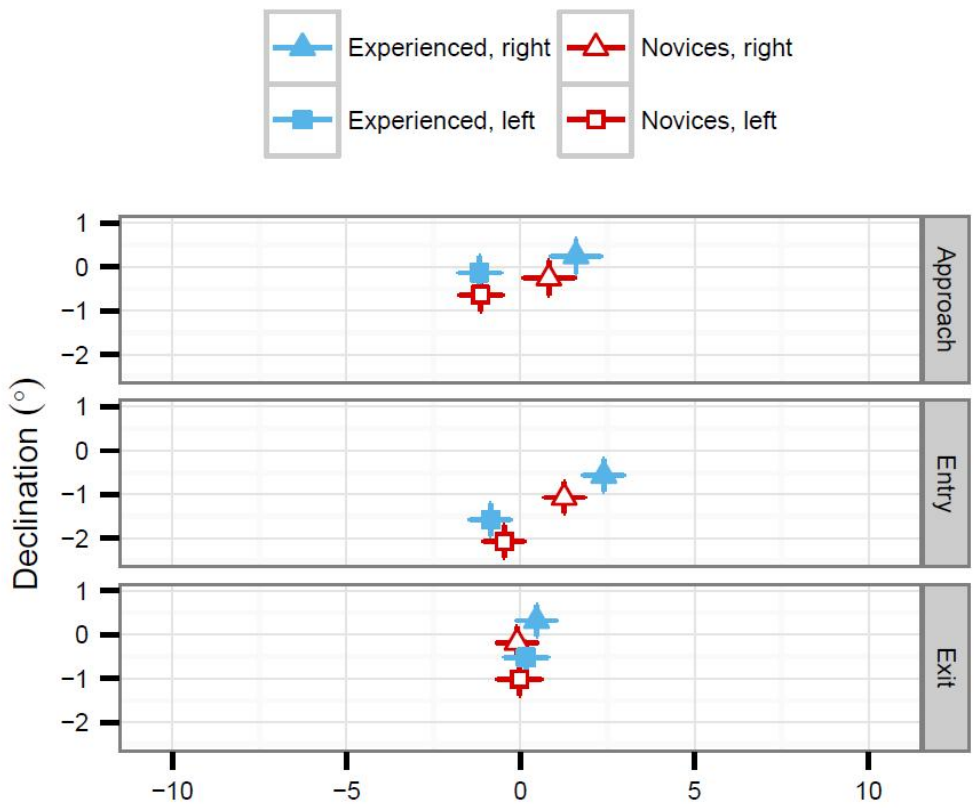

Eccentricity relative to $2 \mathrm{~s}$ time-headway point $\left({ }^{\circ}\right)$

(d)

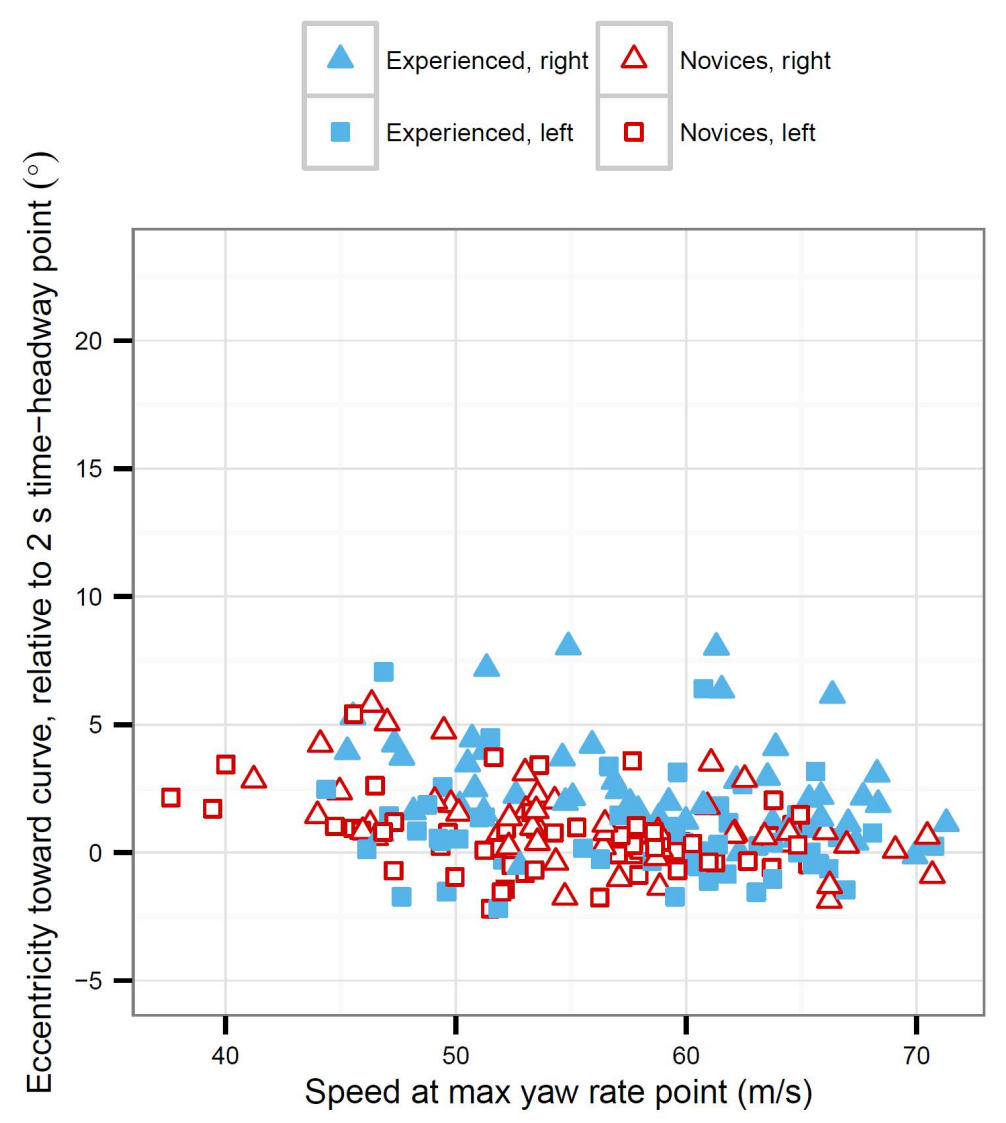


Fig. 5. Horizontal and vertical modes in vehicle centred and $2 \mathrm{~s}$ time-headway direction (TH2) centred coordinates. a) Model predicted means over curves in vehicle centred coordinates, separately for experience group, curve phases and curve direction. Error bars show $95 \%$ confidence intervals. b) Dependence of the vehicle centred horizontal mode ( $y$-scale, positive values to left in left-hand curves and to right in right-hand curves) on the vehicle speed ( $x$-scale). Modes are calculated from the entry phase, separately for each curve, direction, and participant, collapsing runs. Speed is the participant's average speed at the maximum yaw rate point over the four runs through the curve, directions separately. c) Model predicted means over curves in TH2 centred coordinates, separately for experience group, curve phases, and curve direction. Error bars show 95 $\%$ confidence intervals. d) Dependence of the TH2. centred horizontal mode ( $y$-scale, positive values to left in left-hand curves and to right in right-hand curves) on the vehicle speed (x-scale). Modes are calculated from the entry phase, separately for each curve, direction and participant, collapsing runs. Speed is the participant's average speed at the maximum yaw rate point over the four runs.

\subsection{Horizontal and vertical dispersion}

Look-ahead fixations should result in larger horizontal dispersion. The above results show the TH2 reference direction is a good estimate for guiding fixations toward the road-ahead, effectively controlling speed differences, both between curves and driver groups. Consequently, we will use $\mathrm{TH} 2$ centred fixation eccentricities in the examination of horizontal dispersion.

From the TH2 centred data, we calculated the standard deviation of the horizontal eccentricities. The dispersion decreased linearly from the approach to exit phase $(F(2,338)=67.42, p<0.001)$. Experienced drivers had larger dispersion with marginal significance $(F(1,16)=3.83, p=0.068)$. Curve direction and curve phase had a significant interaction $(F(2,338)=5.95, p=0.003)$, which shows that the decrease in horizontal deviation from approach to exit was larger in right curves compared to left curves. However, the density estimates suggest that the main difference between the novice and experience drivers could be in the tail of the distribution, consisting of the lookahead fixations over the curve. These look-ahead fixations are often so few that the standard deviation may not be sensitive to differences in them.

Vertical dispersion was small after the fixations to the dashboard and mirrors were excluded. Experienced drivers had a larger dispersion but the effect was far from significant $(p>0.3)$. The only significant effect was the interaction of curve direction and curve phase $(F(2,338)=5.04$, $p=0.007)$, indicating increase of vertical dispersion in the exit phase of left curves. However, the effect is not very clear and can be due to geometrical differences in the curves. We also calculated the vertical dispersion with the fixations to dashboard and mirrors. In this case, in the entry phase where fixations to the dashboard are rare, the vertical dispersion was higher compared to the previous analysis without the fixations to the dashboard and mirrors. However, no robust experience related effects were found. 


\subsection{Road-ahead and look-ahead fixations}

Table 1 shows the percentages of dwell time on road-ahead and look-ahead fixations. Entry, exit and beyond sectors are collapsed to a single look-ahead fixation category. As Fig. 6 demonstrates there is no large variation between curves in the distribution of fixation eccentricities relative to the $2 \mathrm{~s}$ time-headway direction. The road-ahead and look-ahead fixation percentages, especially, do not depend on speed or curvature of an individual curve (see supplementary Figs. 13-16). Experienced drivers had consistently higher proportion of look-ahead fixations and their variance is larger. Therefore, in the following analysis the curve was treated as a random factor.

Table 1. Average dwell time and standard deviation in different sectors and other areas of interest, for curve phases and directions. The look-ahead sector consists of entry, exit and beyond sectors. Percentages were first calculated separately for each driver and each run, then averaged.

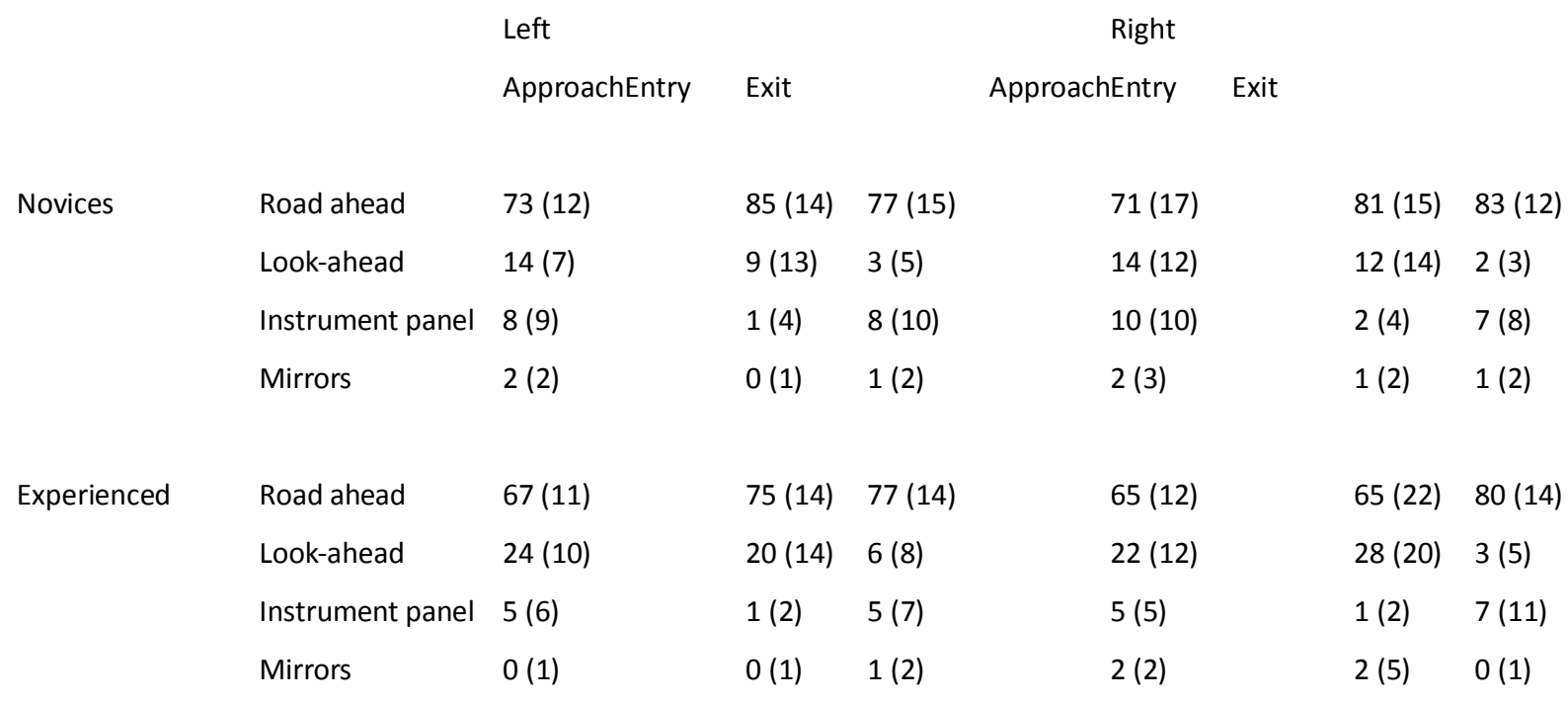



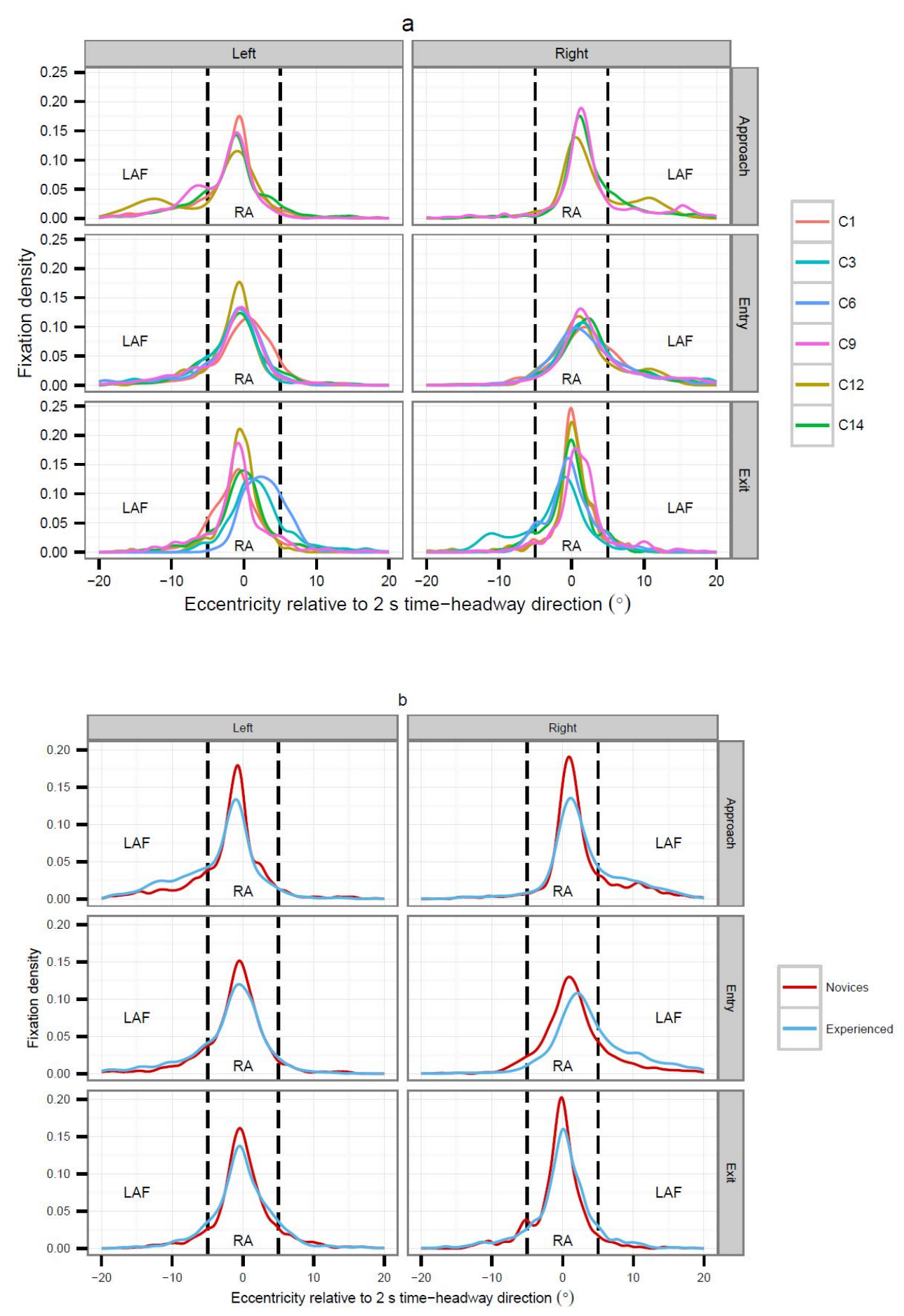

Fig. 6 a). Density estimates of horizontal fixation eccentricity relative to the $2 \mathrm{~s}$ time headway point (TH2) separately for different curves, curve directions and phases. b) Density estimates of horizontal fixation eccentricity relative to the TH2 separately for novice vs. experienced drivers, curve directions and phases. Road-ahead sector (RA) is separated with dashed lines and the direction of look-ahead fixations (LAF) marked. 
As there were very few clearly identifiable look-ahead fixations in the curve exit phase, we included only the approach and entry phases to the statistical model. Experienced drivers had a longer dwell time on the look-ahead sector $(F(1,16)=8.05, p=0.01)$ (Fig. 7a). Interaction of curve direction and the entry phase was significant $(F(1,1049)=31.80, p=0.001)$. In right curves the curve phases had a similar dwell time, but on the left curves dwell time was longer on the approach phase than in the entry phase. Overall, the look-ahead dwell time was longer on approach than in entry phases $(F(1,1049)=37.20,<0.001)$ and longer on left curves than right curves $(F(1$, $197)=5.33, p=0.022)$. There was also a significant decreasing linear trend with run $(F(3,1049)=4.58$, $p=0.034$, from first run's $14.4 \%$ to last run's $10.7 \%$ ).

Experience related difference in look-ahead dwell time can be due to either the number of fixations or the duration of fixations. There was not any substantial difference related to experience on fixation durations. The only significant difference was with curve phase $(F(1$, $759)=12.94, p<0.001$, approach $=265 \mathrm{~ms}$, entry $=242 \mathrm{~ms})$ and curve direction $(F(1,190)=10.04$, $\mathrm{p}=0.002$, right $=241 \mathrm{~ms}$, left $=266 \mathrm{~ms}$ ). Experienced drivers had larger look-ahead fixation rate (number of look-ahead fixations per curve phase duration) $(F(1,16)=8.63, p=0.010$, novices $=0.11$ freq $/ \mathrm{s}$, experienced $=0.25 \mathrm{freq} / \mathrm{s})$, especially in entry phases of the right curves $(F(1,1049)=109.60$, $\mathrm{p}<0.001)$. Also, the look-ahead fixation rate had a linearly decreasing trend over runs $(F(3$, $1049)=6.13, p<0.004$, from first run's $0.21 \mathrm{freq} / \mathrm{s}$ to last run's $0.15 \mathrm{freq} / \mathrm{s})$. Together, look-ahead fixation duration and rate measures indicate that differences in look-ahead dwell times are due to a fewer number of look-ahead fixations, rather than to their shorter duration.

Road-ahead dwell time results were largely opposite to the look-ahead dwell time results (Fig. 7b). Experienced drivers had shorter dwell times on the road ahead sector than novices $(F(1,16)=4.76$, $p=0.044)$. Interaction of curve direction and curve phase was significant $(F(1,1048)=14.05$, $p<0.001$ ), indicating that in entry phase of the left curves, the road ahead sector was looked at markedly more. The main effect of curve phase $(F(1,1048)=103.67, p<0.001)$ showed an increase of road ahead sector from approach to entry phase. Interestingly, there was also a significant interaction of curve phase and experience $(F(1,1048)=6.41, p=0.011)$, showing that the experience related difference was more pronounced in the entry phase than approach phase. There was also significant increasing linear trend with run $(F(3,1048)=2.76, p=0.041)$. 


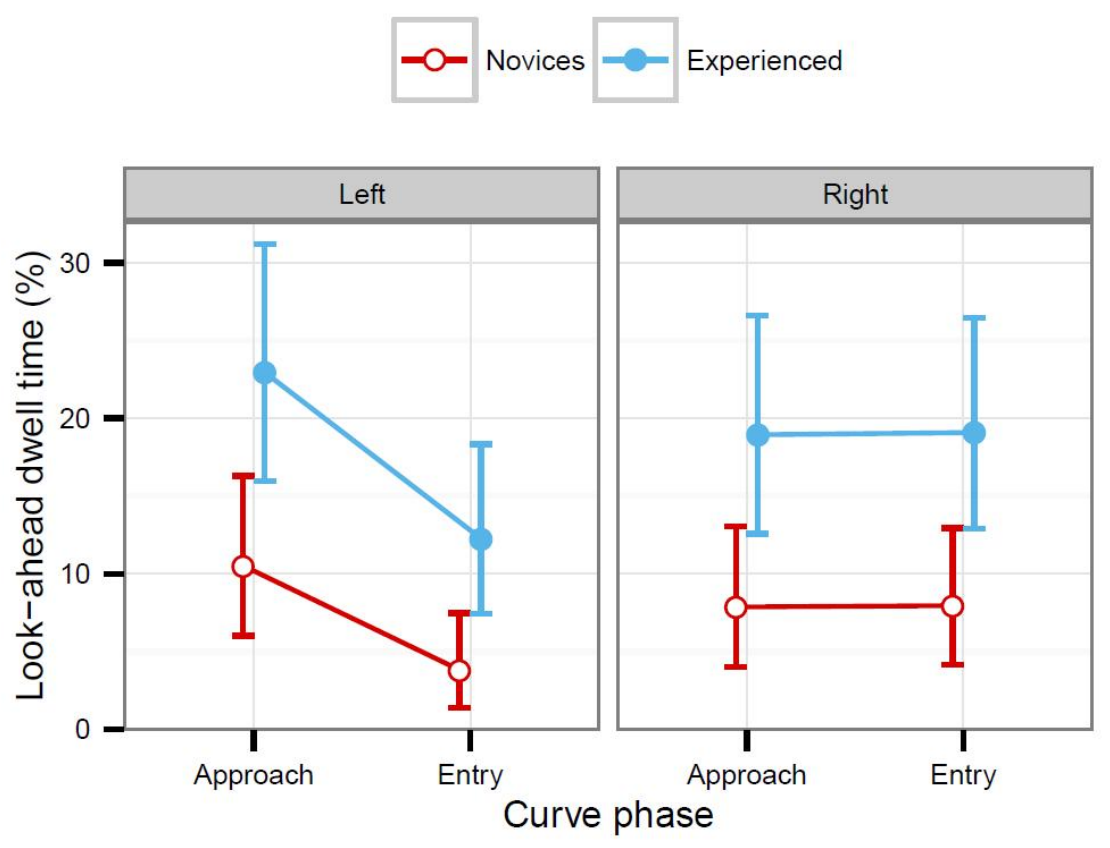

b

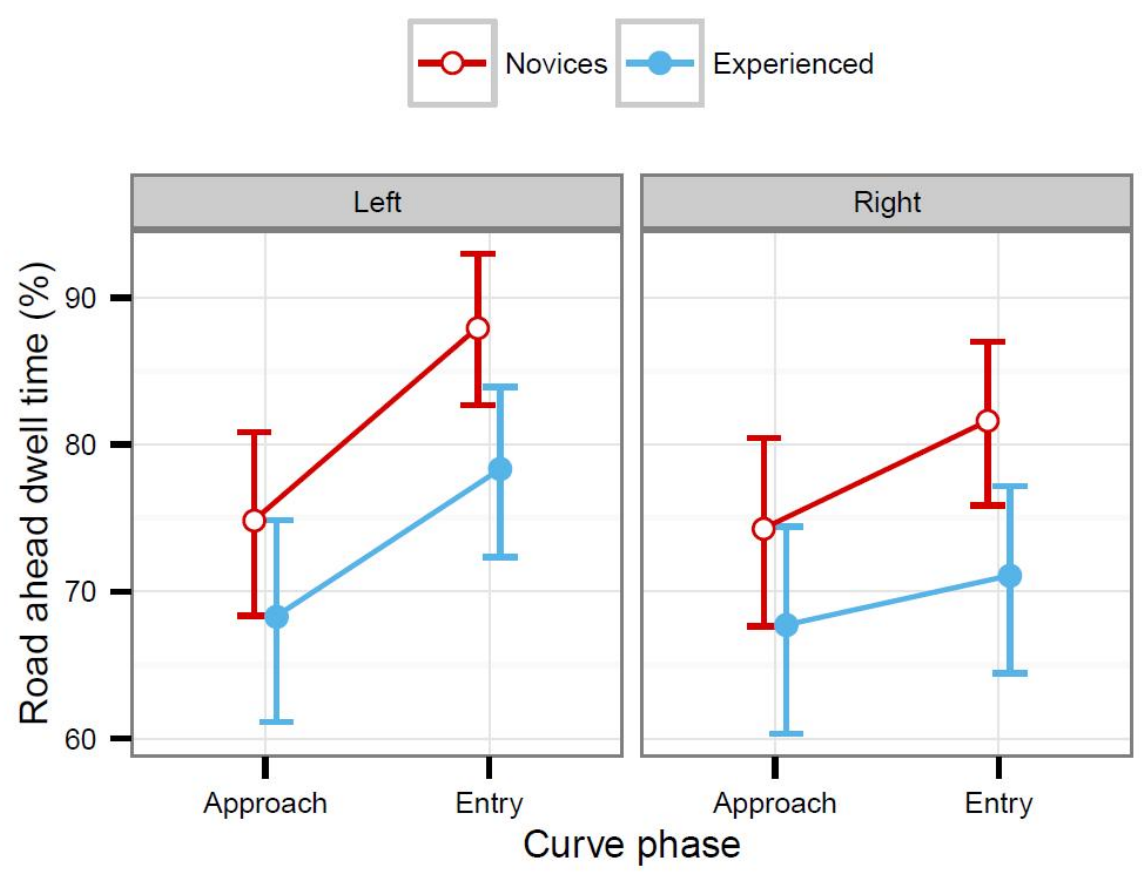

Fig. 7. a) Model predicted percentage of look-ahead dwell time in approach and entry phases, separately for left and right curves and novice and experienced drivers. $95 \%$ confidence intervals are marked. b) Model predicted percentage of road-ahead dwell time in approach and entry phases, separately for left and right curves and novice and experienced drivers. $95 \%$ confidence intervals are marked. 


\subsection{Origins of the look-ahead fixations}

We also compared modes and standard deviations of the look-ahead fixation origin location distributions (Fig. 8) between curves. Modes and standard deviations were calculated for each curve, separately for novice and experienced driver groups. Considering the curves where both approach and entry phases were present, we see that the look-ahead fixations are done during both phases, but the mode of the origin location distributions are consistently near the entry point of the curve, for both groups $(r=0.91)$. No significant difference between the groups in the modes or in the standard deviation was found when tested with a t-test.
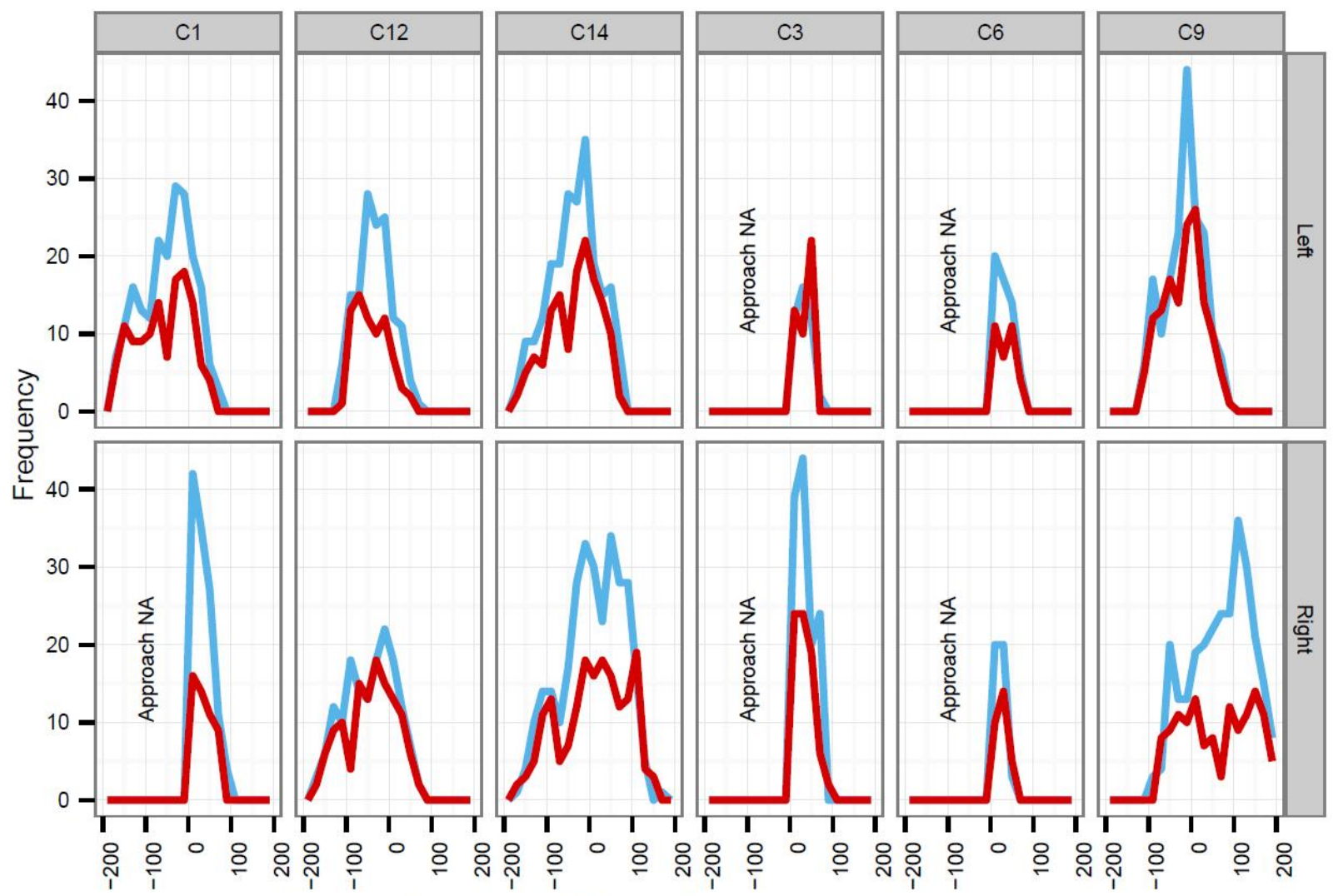

Origin point relative to the curve entry point $(\mathrm{m})$

Fig. 8. Look-ahead fixation origin locations relative to the curve entry point, separately for different curves, curve directions and experience groups. The curves which had no straight approach phase have been marked and no fixations from the approach are included. 


\subsection{Targets of look-ahead fixations}

Density estimates as well as figures with fixations classified to different sectors (see supplementary material) suggest that in general, look-ahead fixations do not target any specific curve phase.

Rather, their distribution appears to depend more on the eccentricity required to make them: lookahead fixations with small eccentricity relative to the $\mathrm{TH} 2$ direction are more common than lookahead fixations with high horizontal eccentricity (Fig. 6).

Eccentricity dependence is supported by an analysis of dwell time of fixations targeting the exit and beyond sectors in approach and entry phases: due to different road geometry, the eccentricity of the maximum yaw rate point is different in different curves. We modelled the effect of a curve using the eccentricity of the maximum yaw rate point in the entry point as a covariate. (In this case, the curve was not included as a random factor.) We found that the eccentricity of the maximum yaw rate at the entry point strongly predicted dwell time of fixations toward the exit and beyond phases of the curve (slope $=-0.48, \mathrm{SE}=0.10, \mathrm{~F}(1,322)=24.541, \mathrm{p}<0.001$ ) (Fig. 9). In the model, effect of experience was also significant, novices having smaller dwell time over the maximum yaw rate point $(F(1,16)=8.511, p=0.010)$.

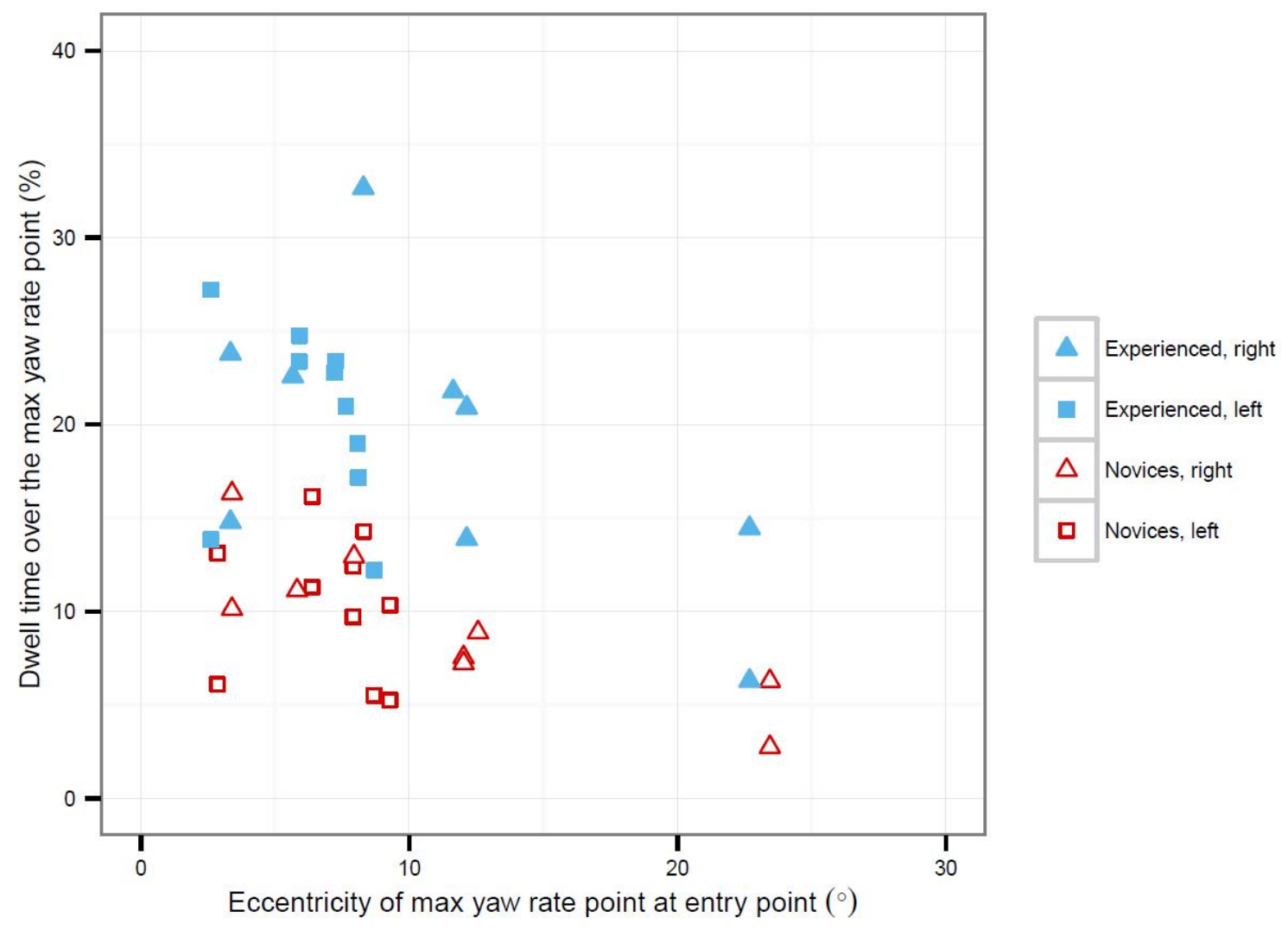

Fig. 9. Percentage of look-ahead fixations over the maximum yaw rate point (exit and beyond sectors) as a function of the average horizontal eccentricity of the maximum yaw rate point at the curve entry point. The approach and entry phases were collapsed. Each point is the average of values within each curve, separately for novice and experienced drivers. Fixations from the approach and entry phases were collapsed. 


\subsection{Steering and speed}

Steering performance was quantified in the entry and exit phases as the high frequency component of the yaw rate signal. We subtracted the smoothed yaw rate used in the curve phase detection (2nd order Savitzky-Golay with $2 \mathrm{~s}$ window) from the yaw rate signal. Average deviation (average of absolute differences) was used as a measure for smoothness, and is used to quantize the high-frequency fluctuation of the yaw rate (the smaller the smoother).

Novice drivers' steering was less smooth in the exit phase of the right curves (phase $\mathrm{x}$ direction $\mathrm{x}$ experience $F(1,1496)=11.405, p<0.001, M=0.36 \%, S D=0.11$ vs. $M=0.28 \% s, S D=0.08$, Cohen's $\mathrm{d}=0.84$ ), but otherwise there were no differences in steering smoothness.

Speed was calculated at curve entry, maximum yaw rate and exit points (Fig. 10). Overall, novices drove slower than experienced drivers $(F(1,16)=4.881, p=0.042)$. Speed increased linearly with every run $(F(3,2343)=50.959, p<0.001)$, and the increase was more marked with novice drivers (experience $x$ run, $F(3,2343)=4.826, p=0.002$ ). Regarding the curve measurement points, experienced drivers' exit point speed was, but novice drivers' was not, significantly higher than their maximum yaw rate speed (location $x$ experience $F(2,2343)=20.453, p<0.001$ ).

We also calculated the speeds 2, 4 and $6 \mathrm{~s}$ before the curve entry point in order to investigate whether the experienced drivers slow down in anticipatory fashion before the curve entry point. We found no significant difference between novice and experienced drivers in the change of speed between any of these locations and the entry point.

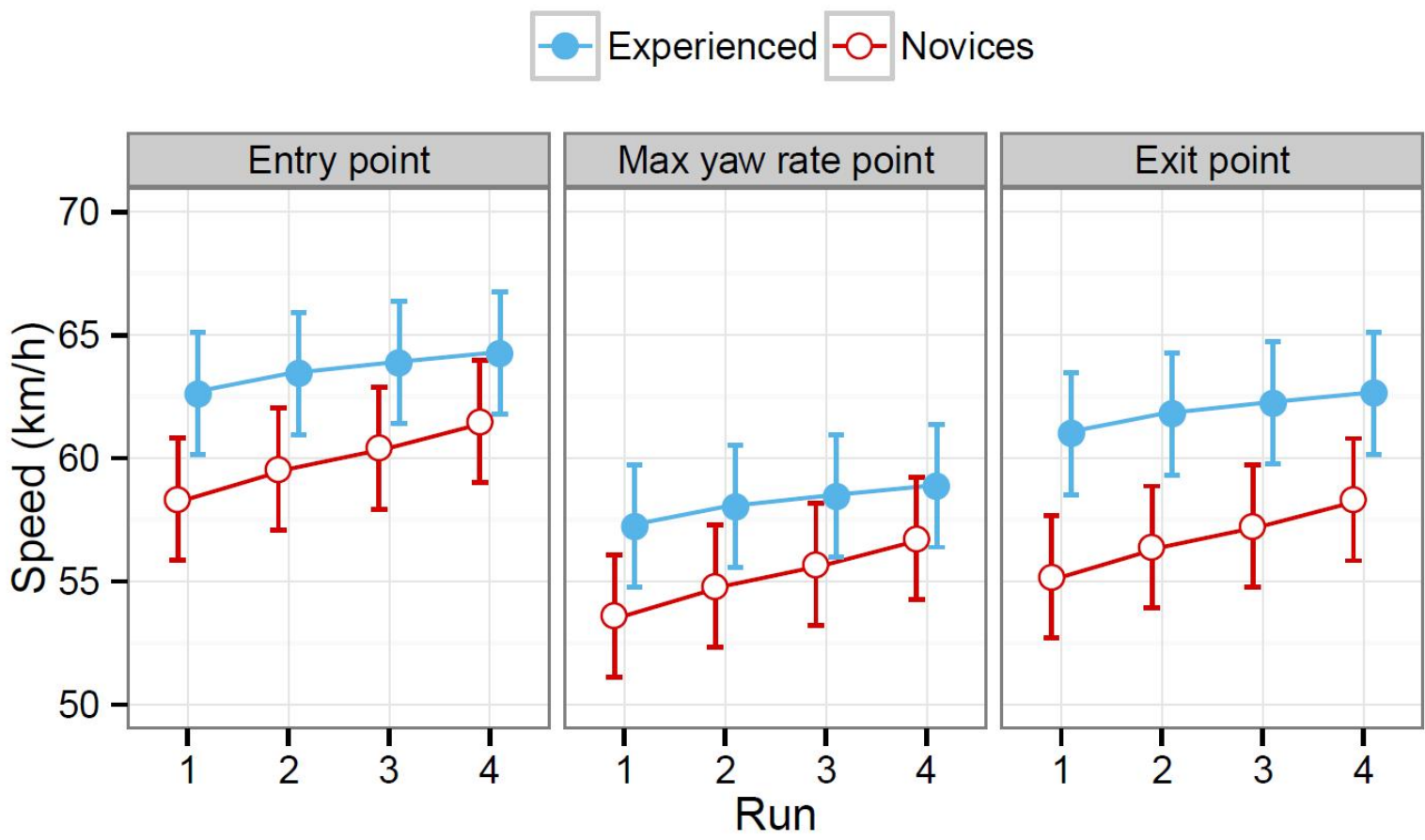

Fig. 10. Model predicted speeds at the curve entry, maximum yaw rate and exit points, separately for experience groups. $95 \%$ confidence intervals are marked. 


\section{Discussion}

When steering a vehicle in curves on rural road, drivers look mostly toward the road-ahead with a small anticipatory preview (e.g. Land and Lee, 1994; Mars, 2008; Lappi et al., 2013). This gaze behaviour is predicted by many current steering models, which are based on the assumption that drivers foveally monitor a steering point (Wann and Land, 2000) for online control of steering and use peripheral vision to support lane-keeping (e.g. Salvucci and Gray, 2004; Summala et al., 1996; Mourant and Rockwell, 1972).

We postulated that there is also a higher control level of trajectory planning, which can influence steering (Cavallo et al., 1988) and speed choice (e.g. Charlton, 2007; Cruzado and Donnell, 2009). On a straight road, visual information for planning the trajectory can be partly obtained from the same road-ahead direction, for example from the entry of the curve, while the road further up in the curve is so eccentric that drivers need to make eccentric look-ahead fixations away from the steering point in order to foveate it (Lehtonen et al. 2012, 2013; Mars and Navarro 2012). During these look-ahead fixations, foveal vision is disengaged from the visual guidance of online steering control, and presumably used for constructing and updating the trajectory plan. Therefore lookahead fixation dwell time can be used as an index of trajectory planning.

In the present on-road study we investigated the effect of driving experience on allocation of gaze between the road-ahead and look-ahead fixations when approaching and negotiating curves with an open view over the curve on a rural road. We identified fixations which were less than 5 degrees from the $2 \mathrm{~s}$ time-headway direction as road-ahead fixations. These road-ahead fixations should contain the guiding fixations serving the online control of steering. Fixations which were more eccentric and targeting the road further ahead were identified as look-ahead fixations, serving the trajectory planning rather than the online control of steering.

We observed look-ahead fixations both in the approach and entry phases of the curves. In the exit phase, look-ahead fixations were very few because most of the road is visible in the direction of locomotion. Look-ahead fixation dwell time was longer in the approach than in the entry phase. An opposite pattern was found for road-ahead dwell time. This probably reflects the visual demands of online control of steering. When negotiating a curve, visual demand of steering control is higher than when approaching a curve on a straight road (Tsimhoni et al., 2001). Because online steering control has a higher priority than trajectory planning in the control of driving, it is prioritized in gaze allocation (Recarte and Nunes, 2003).

The above interpretation is consistent with the recent result by Mars and Navarro (2012) who demonstrated that when the need to actively steer was removed in a simulator, look-ahead fixations increased. Removal of active steering could be interpreted to reduce visual demand of the guidance and stabilising levels of steering, making it possible to allocate visual attention to trajectory planning. Similarly, Mennie et al. (2007) suggested that look-ahead fixations in a sequential manipulation task were most typical when the completion of the current phase did not require visual guidance.

We also found that dwell time on look-ahead fixations was longer in right-hand curves, where the vehicle A-frame is not occluding the curve further ahead. This suggests that visibility in general is probably a factor affecting the look-ahead fixations.

Compared to novices, experienced drivers had shorter road-ahead dwell time both when approaching and entering curves even though they drove faster than novices. Therefore, the 
current results corroborate the results by Summala (1998) and Summala et al. (1996) that experienced drivers are better able to use their peripheral vision for steering control. Furthermore, the experienced drivers had longer look-ahead fixation dwell time than novices. The effect was due to novice drivers' fewer number of look-ahead fixations per second.

The results are in accordance with the interpretation that look-ahead fixations over curves serve trajectory planning. Eccentric look-ahead fixations are cognitively costly (Wickens et al., 2009; Lehtonen et al., 2013) and are unlikely to be performed without a purpose. Experienced drivers are considered better in their anticipation skills (Jackson et al., 2009; Underwood, 2007), and thus it is reasonable to suppose that this also applies to the trajectory planning. More complex trajectory planning creates a greater demand for visual information from the future road, resulting in more frequent look-ahead fixations. Simultaneously, experienced drivers' better ability to utilize peripheral vision for steering (Summala et al., 1996) leaves them more free gaze time to invest on the trajectory planning.

Further support for the link between trajectory planning and look-ahead fixations comes from the effect of run on the look-ahead fixations. Look-ahead fixation dwell time had a significant linearly decreasing trend over runs. This suggests that drivers could be creating a mental model of the environment. In other words, drivers need less visual guidance for their trajectory planning if the previous memorized plans are to be utilized. On the other hand, decrease in look-ahead fixations can be due to increasing speed over runs, which probably increased the visual demand of steering, or due to fatigue.

In the current naturalistic study it is difficult to directly evaluate experienced and novice drivers' trajectory plans. If the trajectory plan is wrong, the problems manifest typically after entering the curve. The experienced drivers had significantly smoother yaw rate in the exit phase in the left curves. Also, the experienced drivers accelerated faster during the exit phase of the curve, which was interpreted as more forward oriented driving and is linked to better planning.

The simulator experiment by Muttart et al. (2013) showed that experienced drivers had a larger speed drop when approaching a sharp curve, which could be interpreted to indicate more anticipatory speed regulation due to better trajectory planning skills. In the current study, there were no differences in anticipatory speed regulation between novices and experienced drivers. It is possible that because all the curves had an open view over the curve (including the curves without a straight approach phase), the drivers started to adapt their speed even before the $6 \mathrm{~s}$ we investigated. Also, it is possible that the speed limit (70 km/h, which drivers typically respected) was so low that there was little need to brake before the entry phase.

A limitation of the current naturalistic study is that it was not feasible to distinguish between anticipation of road geometry and oncoming cars. Previous studies have shown that look-ahead fixations occur also when there are no other road users (Lehtonen et al., 2012, 2013), and anticipatory eye movements in curves may help to avoid crashes (Muttart et al., 2013).

Modes of the fixations' horizontal and vertical eccentricity distributions suggest that novice drivers concentrate their guiding fixations slightly closer along the road than experienced drivers while driving in curves. Such interpretation is in-line with Mourant and Rockwell's (1972) controversial finding (see Falkmer and Gregersen, 2005) that novices look closer. However, the difference is mainly due to the horizontal component and largely disappears if the speed is controlled using eccentricity of the $2 \mathrm{~s}$ time-headway point as the reference. This result is consistent with the future path models (Wann and Swapp, 2000; Wilkie et al., 2008) and in accordance with the hypothesis of 
visual buffer of 1-2 s (Land and Furneaux, 1997; Mars, 2008; Vansteenkiste et al., 2013).

We did not find any significant difference in the vertical variability of the fixations' eccentricities, which would have indicated that novice drivers more often foveated the near zone (cf. Summala et al., 1996). We excluded fixations to the instrument panel and to the mirrors, which may explain the discrepancy of the vertical dispersion results to those found by Crundall and Underwood (1998). As such, the current results do not suggest that novice drivers $(<5000 \mathrm{~km})$ would use their foveal gaze for control of the stabilizing level of steering, e.g. the lane-position.

The horizontal eccentricity distributions of fixations were highly similar across curves, even though the eccentricity of the curve exit point of the trajectory varied strongly between curves. There were fewer fixations for higher eccentricities. This is consistent with suggestions that larger shifts in attentional focus involve higher cognitive cost, explaining why they are executed less often (Wickens et al., 2009). However, in curve C12 where the exit point was located at a small eccentricity, the look-ahead fixations appeared to concentrate near the direction of the exit point (see supplementary Figs. 1 -12). Horizontal dispersion measure reflected look-ahead dwell time results, suggesting that look-ahead fixations over curves are one component of larger horizontal variability often linked to experienced drivers (Mourant and Rockwell, 1972; Falkmer and Gregersen, 2005).

Overall, the difference between the novices and experienced drivers on look-ahead fixation behaviour appeared to be more in the quantity than in quality. However, the difference in the level of experience is not very large between the groups we studied, and it is possible that pattern of look-ahead fixations would be substantially different for learner drivers and expert drivers. For example, professional race drivers need to make complex and detailed trajectory plans in order to minimise their lap times. Land and Tatler (2001) studied eye movement of a professional formula driver on a track, and found that the driver typically fixated very far, through the curves. It is possible that for such a driver the online steering control is almost completely peripheralized and trajectory planning on a race circuit is largely memory based.

\subsection{Automatization, attention and look-ahead fixations}

The effect of driving experience on look-ahead fixations can be explained from the perspective of automatization. When the online control of steering becomes more automatized, it requires less attention (Fitts and Posner, 1967; Shiffrin and Schneider, 1977; Horrey et al., 2006). Consequently, spare attention can be allocated to higher levels of the task, including trajectory planning. This change is reflected in the eye movements as increasing look-ahead fixations. In other words, the eye movements become more future oriented (cf. Sailer et al., 2005). Similarly, when the steering task itself is easier, e.g. due to road geometry (Tsimhoni et al., 2001) or automatic steering (Mars and Navarro, 2012), spare attention can be invested to look-ahead fixations.

However, such interpretation treats attention as a unitary resource which is shared between components of the task (the modal view of attention, Logan, 1988). It does not recognize that there can be multiple, parallel resources available for the driver (Horrey et al., 2006; Wickens et al., 2009). For a complete explanation, it would be necessary to differentiate between peripheral and foveal visual processing demands at the different levels of steering, and also examine executive functions which are likely needed for shifts of attention between look-ahead and guiding fixations (Lehtonen et al., 2012). 


\subsection{Trajectory planning and road safety}

The road environment and other road users create both static and dynamic constraints (goals that need to be fulfilled or obstacles that need to be avoided) for locomotion (Gibson and Crooks, 1938; Fajen and Warren, 2003; Summala, 2007). Trajectory planning can be seen as process of satisfying these constraints. Drivers have goals and motives which push the driver to choose trajectories which are fast and smooth enough (Summala, 2007), which can be also seen as soft constraints for the trajectory plan, compared to hard constraints such road edges and other road users. When trajectory planning skills are not well developed, motives like a desire for a high speed, may be given too much weight in decisions because the environmental constraints are not correctly recognized. Therefore novice drivers' poorer trajectory planning skills can be a contributing factor for single vehicle accidents in curves.

\section{Acknowledgement}

We thank Liisa Hintikka, Henri Kotkanen and Andres Levitski for participation in the data collection and analysis. Jami Pekkanen contributed to computer code used in the analysis. Caitlin Dawson proofread the manuscript. Finnish Cultural Foundation (grant no. 00130510) and Eteläsuomalaisten ylioppilaiden säätiö are gratefully acknowledged for grants for the first author. 
References

Abdel-Aty, M. A., \& Radwan, A. E. (2000). Modeling traffic accident occurrence and involvement, Accident Analysis and Prevention, 32, 633-642.

Ahlström, C., Victor, T., Wege, C., \& Steinmetz, E. (2012). Processing of eye/head-tracking data in large-scale naturalistic driving data sets. IEEE Transactions on Intelligent Transportation Systems, 13(2), 553-564.

Ballard, D. H., Hayhoe, M. M., \& Pelz, J. B. (1995). Memory representations in natural tasks. Journal of Cognitive Neuroscience, 7(1), 66-80. doi:10.1162/jocn.1995.7.1.66

Bernardin, D., Kadone, H., Bennequin, D., Sugar, T., Zaoui, M., \& Berthoz, A. (2012). Gaze anticipation during human locomotion. Experimental Brain Research, 223(1), 65-78. doi:10.1007/s00221-012-3241-2

Boer, E. R. (1996). Tangent Point Oriented Curve Negotiation. In: Intelligent Vehicles Symposium. IEEE.

Cavallo, V., Brun-Dei, M., Laya, O., \& Neboit, M. (1988). Perception and anticipation in negotiating curves: the role of driving experience. In A. G. Gale, M. H. Freeman, C. M. Haslegrave, P. Smith, \& S. P. Taylor (Eds.), Vision in Vehicles II (pp. 365-374). Amsterdam: North-Holland.

Charlton, S. G. (2007). The role of attention in horizontal curves: a comparison of advance warning, delineation, and road marking treatments. Accident Analysis and Prevention, 39(5), 873-885. doi:10.1016/j.aap.2006.12.007

Clarke, D. D., Ward, P., Bartle, C., \& Truman, W. (2006). Young driver accidents in the UK: the influence of age, experience, and time of day. Accident Analysis and Prevention, 38(5), 871878. doi:10.1016/j.aap.2006.02.013

Cloete, S., \& Wallis, G. (2011). Visuomotor control of steering: the artefact of the matter. Experimental brain research, 208(4), 475-89. doi:10.1007/s00221-010-2530-x

Cohen, A. S., \& Studach, H. (1977). Eye movements while driving cars around curves. Perceptual and motor skills, 44(3), 683-689.

Cooper, R., \& Shallice, T. (2000). Contention scheduling and the control of routine activities. Cognitive Neuropsychology, 17(4), 297-338. doi: 10.1080/026432900380427

Crundall, D. E., \& Underwood, G. (1998). Effects of experience and processing demands on visual information acquisition in drivers. Ergonomics, 41(4), 448-458.

Cruzado, I., \& Donnell, E. T. (2010). Factors affecting driver speed choice along two-lane rural highway transition zones, Journal of Transportation Engineering, 136(8), 755-764. doi: 10.1061/(ASCE)TE.1943-5436.0000137

Donges, E. (1978). A two-level model of driver steering behavior. Human Factors, 20(6), 691-707.

Fajen, B. R., \& Warren, W. H. (2003). Behavioral dynamics of steering, obstable avoidance, and route selection. Journal of Experimental Psychology: Human Perception and Performance, 29(2), 343-362. doi:10.1037/0096-1523.29.2.343

Falkmer, T., \& Gregersen, N. P. (2005). A comparison of eye movement behavior of inexperienced and experienced drivers in real traffic environments. Optometry and vision science, 82(8), 732739. 
Fitts, P. M., \& Posner, M. I. (1967). Human Performance. Brooks/Cole Publishing Company. Belmont, California.

Frissen, I., \& Mars, F. (2013). The effect of visual degradation on anticipatory and compensatory steering control. QuarterlyJjournal of Experimental Psychology (2006), 37-41. doi:10.1080/17470218.2013.819518

Gibson, J.J. \& Crooks, L.E. (1938). A theoretical field-analysis of automobile driving. American Journal of Psychology, 51, 453-471.

Godthelp, H., Milgram, P., \& Blaauw, G. J. (1984). The development of a time-related measure to describe driving strategy. Human Factors, 26(3), 257-268. doi:10.1177/001872088402600302

Grafton, S. T., \& Hamilton, A. F. de C. (2007). Evidence for a distributed hierarchy of action representation in the brain. Human Movement Science, 26(4), 590-616.

Hassan, Y., \& Sarhan, M. (2012). Operational effects of drivers misperception of horizontal curvature. Journal of Transportation Engineering, 138(11), 1314-1320. doi:10.1061/(ASCE)TE.1943-5436.0000436

Hayhoe, M. M., Shrivastava, A., Mruczek, R., \& Pelz, J. B. (2003). Visual memory and motor planning in a natural task. Journal of Vision, 3(1), 49-63.Horrey, W. J., Wickens, C. D., \& Consalus, K. P. (2006). Modeling drivers' visual attention allocation while interacting with invehicle technologies. Journal of ExperimentalPpsychology. Applied, 12(2), 67-78. doi:10.1037/1076-898X.12.2.67

Imai, T., Moore, S. T., Raphan, T., \& Cohen, B. (2001). Interaction of the body, head, and eyes during walking and turning. Experimental Brain Research, 136(1), 1-18. doi:10.1007/s002210000533

Jackson, L., Chapman, P. \& Crundall, D., (2009). What happens next? Predicting other road users' behaviour as a function of driving experience and processing time. Ergonomics, 52(2), 154164. doi: $10.1080 / 00140130802030714$

Laapotti, S., \& Keskinen, E. (1998). Differences in Fatal Loss-of-control accidents between young male and female drivers. Accident Analysis \& Prevention, 30(4), 435-442.

Land, M. F., \& Furneaux, S. (1997). The knowledge base of the oculomotor system. Philosophical Transactions of the Royal Society of London. Series B, Biological Sciences, 352(1358), 12311239. doi:10.1098/rstb.1997.0105

Land, M. F., \& Lee, D. N. (1994). Where we look when we steer. Nature, 369(6483), 742-744. Retrieved from http://dx.doi.org/10.1038/369742a0

Land, M. F., \& Tatler, B. W. (2001). Steering with the head: the visual strategy of a racing driver. Current Biology, 11(15), 1215-20.

Land, M., \& Horwood, J. (1995). Which parts of the road guide steering? Nature, 377(6547), 33940. doi:10.1038/377339a0

Land, M., \& Horwood, J. (1996). The relations between head and eye movements during driving. In A. G. et al. Gale (Ed.), Vision in Vehicles V (pp. 153-160). Elsevier.

Land, M., Mennie, N., \& Rusted, J. (1999). The roles of vision and eye movements in the control of activities of daily living. Perception, 28(11), 1311-1328. doi:10.1068/p2935

Land, M. F. (2009). Vision, eye movements, and natural behavior. Visual Neuroscience, 26(1), 51- 


\section{2. doi:10.1017/S0952523808080899}

Lappi, O., \& Lehtonen, E. (2013). Eye-movements in real curve driving: pursuit-like optokinesis in vehicle frame of reference, stability in an allocentric-reference coordinate system. Journal of Eye Movement Research, 6(1), 1-13.

Lappi, O., Lehtonen, E., Pekkanen, J. J. O., Itkonen, T. (in press). Beyond the tangent point - gaze targets in naturalistic driving. Journal of Vision.

Lehtonen, E., Lappi, O., \& Summala, H. (2012). Anticipatory eye movements when approaching a curve on a rural road depend on working memory load. Transportation Research Part F: Traffic Psychology and Behaviour, 15(3), 369-377. doi:10.1016/j.trf.2011.08.007

Lehtonen, E., Lappi, O., Kotkanen, H., \& Summala, H. (2013). Look-ahead fixations in curve driving. Ergonomics, 56(1), 34-44. doi:10.1080/00140139.2012.739205

Logan G. D. (1988). Toward an instance theory of automatization. Psychological Review, 95:492527. doi:10.1037/0033-295X.95.4.492

Marigold, D. S., \& Patla, A. E. (2007). Gaze fixation patterns for negotiating complex ground terrain. Neuroscience, 144(1), 302-13. doi:10.1016/j.neuroscience.2006.09.006

Mars, F. (2008). Driving around bends with manipulated eye-steering coordination. Journal of Vision, 8, 1-11. doi:10.1167/8.11.10

Mars, F., \& Navarro, J. (2012). Where we look when we drive with or without active steering wheel control. PloS ONE, 7(8), e43858. doi:10.1371/journal.pone.0043858

Mennie, N., Hayhoe, M., \& Sullivan, B. (2007). Look-ahead fixations: anticipatory eye movements in natural tasks. Experimental Brain Research, 179, 427-442. doi:10.1007/s00221-006-0804-0

Michon, J. A. (1985). A critical view of driver behaviour models: what do we know, what should we do? In L. Evans \& R.C Schwing (Eds.) Human Behaviour and Traffic Safety. New York: Plenum Press. pp. $485-520$.

Mourant, R. R., \& Rockwell, T. H. (1972). Strategies of visual search by novice and experimental drivers. Human Factors, 14(4), 325-35.

Muttart. J. W., Fisher, D.L., Pollatsek, A. P. \& Marquard, J. (2013). Comparison of anticipatory glancing and risk mitigation of novice drivers and exemplary drivers when approaching curves. In: Proceedings of the Seventh International Driving Symposium on Human Factors in Driver Assessment, Training, and Vehicle Design. Retrieved Sep 272013 from http://drivingassessment.uiowa.edu/sites/default/files/DA2013/Papers/033_Muttart_0.pdf

Pelz, J. B., \& Canosa, R. (2001). Oculomotor behavior and perceptual strategies in complex tasks. Vision Research, 41(25/26), 3587-96. doi:http://dx.doi.org/10.1016/S0042-6989(01)00245-0

Pinheiro, J. C., \& Bates, D. M. (2000). Linear Mixed-Effects Models: Basic Concepts and Examples. Mixed-Effects Models in S and S-PLUS. Springer New York. doi:10.1007/978-1-4419-0318-1_1

Recarte, M. A., \& Nunes, L. M. (2003). Mental workload while driving: effects on visual search, discrimination, and decision making. Journal of Experimental Psychology: Applied, 9(2), 119137. doi:10.1037/1076-898X.9.2.119

Sailer, U., Flanagan, J. R., \& Johansson, R. S. (2005). Eye-hand coordination during learning of a novel visuomotor task. Journal of Neuroscience, 25(39), 8833-42. 
Salvucci, D. D, \& Gray, R. (2004). A two-point visual control model of steering. Perception, 33(10), 1233-1248. doi:10.1068/p5343

Salvucci, D. D, \& Goldberg, J. H. (2000). Identifying fixations and saccades in eye-tracking protocols. In: Proceedings of the 2000 symposium on Eye tracking research \& applications (pp. 71-78). New York, NY, USA: ACM. doi:10.1145/355017.355028

Shiffrin, R. M., \& Schneider, W. (1977). Controlled and automatic human information processing: II. perceptual learning, automatic attending and a general theory. Psychological Review, 84(2), 127-190.

Shinar, D., McDowell, E. D., \& Rockwell, T. H. (1977). Eye movements in curve negotiation. Human Factors, 8(4), 237-238.

Shinar, D., Rockwell, T. H., \& Maleki, J. A. (1980). The effects of changes in driver perception on rural curve negotiation. Ergonomics, 23(3), 263-275. doi:10.1080/00140138008924739

Spacek, P. (2005). Track Behavior in Curve Areas : Attempt at Typology. Journal of Transportation Engineering, 131(9)(September), 669-677.

Steen, J., Damveld, H. J., Happee, R., Paassen, M. M. Van, \& Mulder, M. (2011). A Review of Visual Driver Models for System Identification Purposes. In: IEEE International Conference on Systems, Man, and Cybernetics (SMC), 2011 (pp. 2093-2100). Anchorage, AK. doi:10.1109/ICSMC.2011.6083981

Summala, H., Nieminen, T., \& Punto, M. (1996). Maintaining lane position with peripheral vision during in-vehicle tasks. Human Factors, 38:442-451. doi:10.1518/001872096778701944

Summala, H. (1997). Hierarchical model of behavioural adaptation and traffic accidents. In T. Rothergatter \& Carbonell V. (Eds.), Traffic and Transport Psychology: Theory and Application. Amsterdam: Pergamon, pp. 41-52.

Summala, H. (1998). Forced peripheral vision driving paradigm: evidence for the hypothesis that car drivers learn to keep in lane with peripheral vision. In A.G. Gale, I.D. Brown, C.M. Haslegrave, \& S.P. Taylor (Eds.), Vision and Vehicles VI (pp. 51-60). Elsevier, pp. 51-60.

Summala, H. (2007). Towards understanding motivational and emotional factors in driver behaviour: comfort through satisficing. In P. C. Cacciabue (Ed.), Modelling Driver Behaviour in Automotive Environments (pp. 189-207). Springer Verlag.

Tsimhoni, O., Green, P., \& Arbor, A. (2001). Visual demand of driving and the execution of displayintensive in-vehicle tasks. Proceedings of the Human Factors and Ergonomics Society (pp. 1586-1590).

Underwood, G. (2007). Visual attention and the transition from novice to advanced driver. Ergonomics, 50(8), 1235-1249. doi: 10.1080/00140130701318707

Wann, J., \& Land, M. (2000). Steering with or without the flow: is the retrieval of heading necessary? Trends in cognitive sciences, 4(8), 319-324.

Wann, J. P., \& Swapp, D. K. (2000). Why you should look where you are going. Nature Neuroscience, 3(7), 647-648. doi:10.1038/76602

Vansteenkiste, P., Cardon, G., D’Hondt, E., Philippaerts, R., \& Lenoir, M. (2013). The visual control of 
bicycle steering: The effects of speed and path width. Accident Analysis and Prevention, 51, 222-227. doi:10.1016/j.aap.2012.11.025

Wickens, C., McCarley, J., \& Steelman-Allen, K. (2009). NT-SEEV: A model of attention capture and noticing on the Flight Deck. Proceedings of the Human Factors and Ergonomics Society Annual Meeting, 53(12), 769-773. doi:10.1177/154193120905301202

Wilkie, R. M., Wann, J. P., \& Allison, R. S. (2008). Active gaze, visual look-ahead, and locomotor control. Journal of Experimental Psychology: Human Perception and Performance, 34(5), 1150-64. doi:10.1037/0096-1523.34.5.1150 
Supplementary material for Lehtonen, E., Lappi, O., Koirikivi, I., Summala, H. Effect of driving experience on anticipatory look-ahead fixations in real curve driving. Accident Analysis \& Prevention

Corresponding author: esko.lehtonen@helsinki.fi

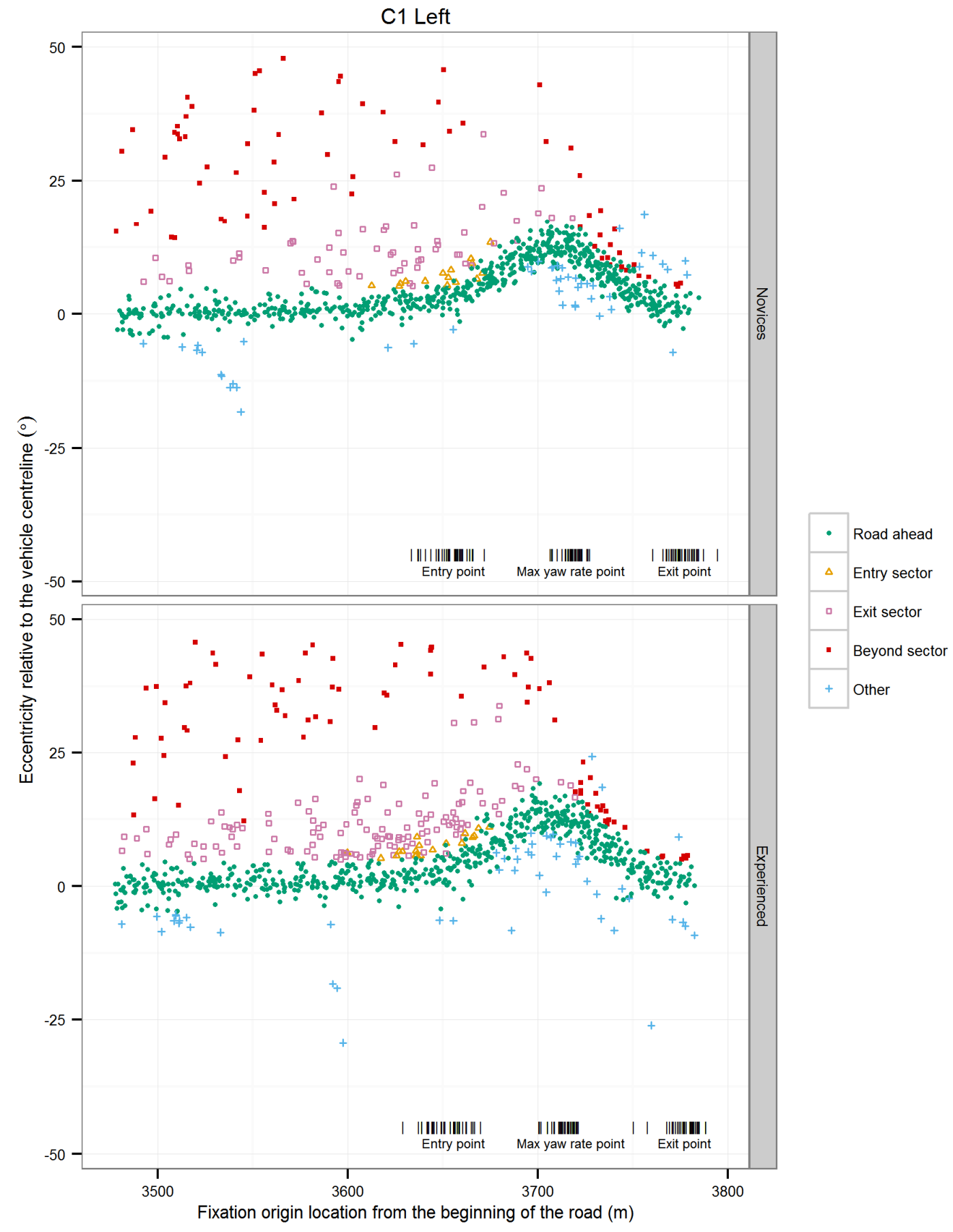

Supplementary Figure 1. Fixations with their origin location ( $x$-scale) and eccentricity relative to the vehicle centreline ( $y$ scale) with a classification (colour and shape), for experience groups (novices, experienced) at curve C1 to left (up). Fixations to road ahead, entry, exit and beyond sectors target the road. Other fixations include those to instrument panels, mirrors, and the scenery. Fixations in different sectors may mix due to differences in individual driving lines, which affect sector boundaries. Distribution of entry, max yaw rate and exit points are marked with vertical lines. 
Supplementary material for Lehtonen, E., Lappi, O., Koirikivi, I., Summala, H. Effect of driving experience on anticipatory look-ahead fixations in real curve driving. Accident Analysis \& Prevention

Corresponding author: esko.lehtonen@helsinki.fi

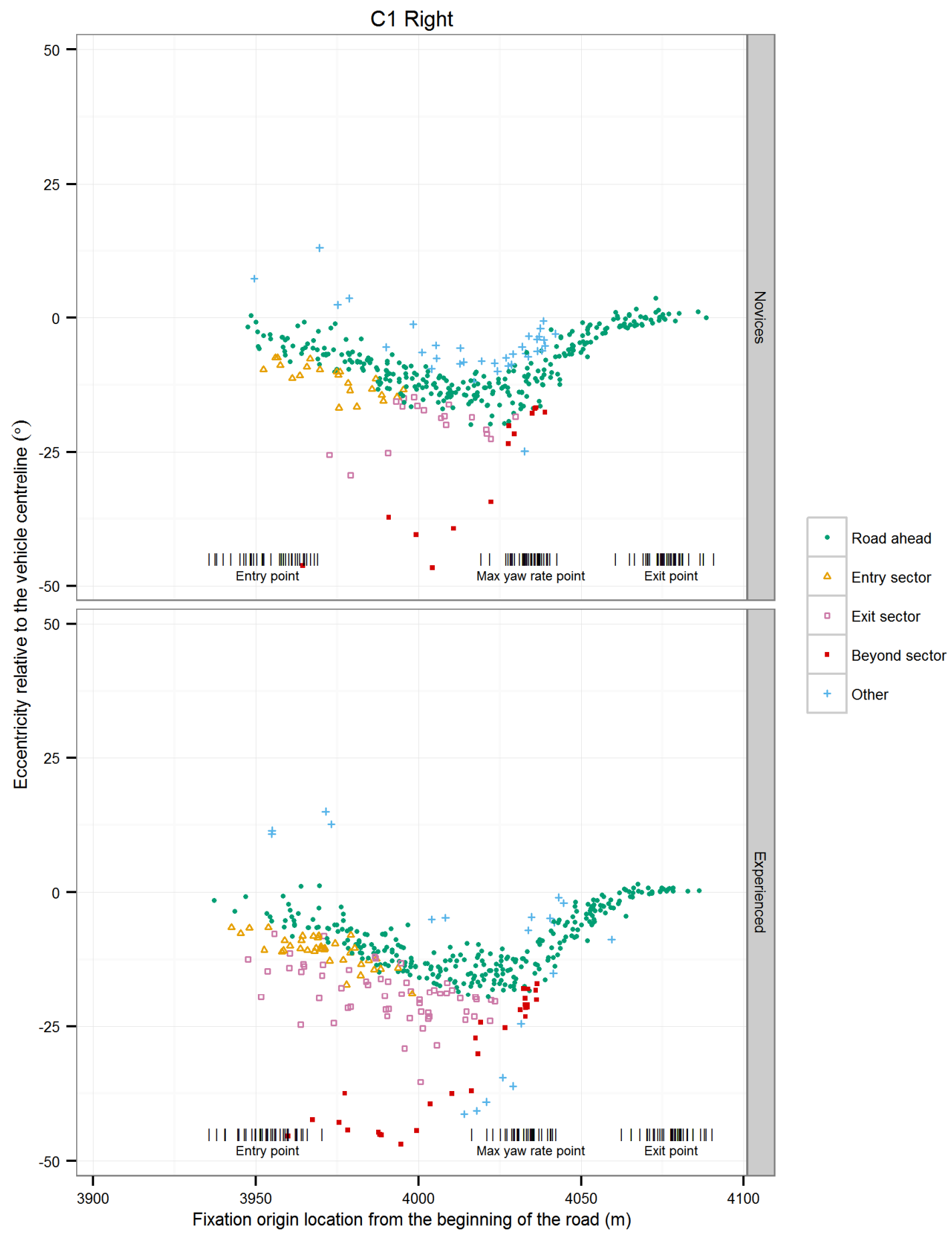

Supplementary Figure 2. Fixations with their origin location ( $x$-scale) and eccentricity relative to the vehicle centreline ( $y$ scale) with a classification (colour and shape), for experience groups (novices, experienced) at curve C1 to right (down). Fixations to road ahead, entry, exit and beyond sectors target the road. Other fixations include those to instrument panels, mirrors, and the scenery. Fixations in different sectors may mix due to differences in individual driving lines, which affect sector boundaries. Distribution of entry, max yaw rate and exit points are marked with vertical lines. 
Supplementary material for Lehtonen, E., Lappi, O., Koirikivi, I., Summala, H. Effect of driving experience on anticipatory look-ahead fixations in real curve driving. Accident Analysis \& Prevention

Corresponding author: esko.lehtonen@helsinki.fi

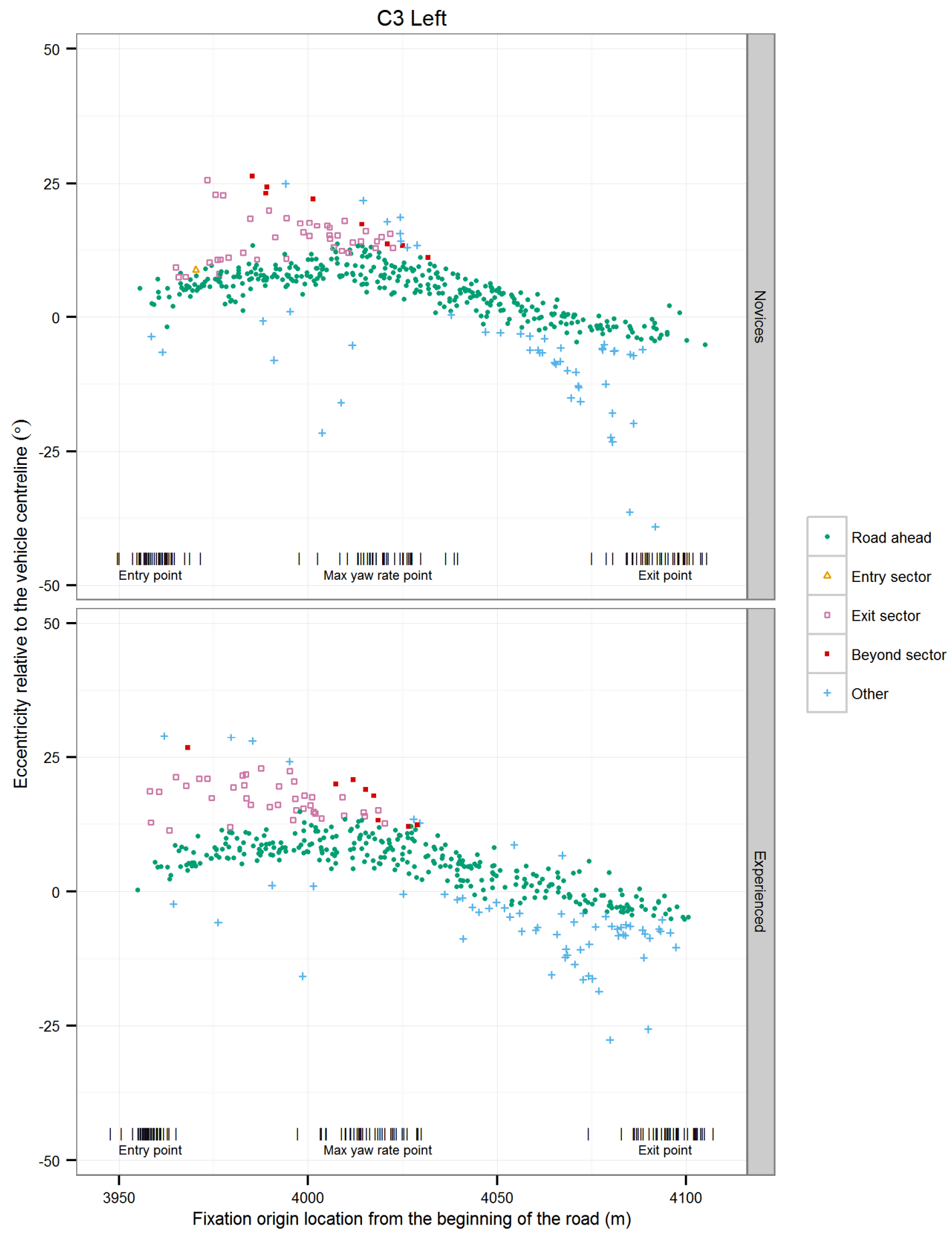

Supplementary Figure 3. Fixations with their origin location ( $x$-scale) and eccentricity relative to the vehicle centreline (yscale) with a classification (colour and shape), for experience groups (novices, experienced) at curve C3 to left (up). Fixations to road ahead, entry, exit and beyond sectors target the road. Other fixations include those to instrument panels, mirrors, and the scenery. Fixations in different sectors may mix due to differences in individual driving lines, which affect sector boundaries. Distribution of entry, max yaw rate and exit points are marked with vertical lines. 
Supplementary material for Lehtonen, E., Lappi, O., Koirikivi, I., Summala, H. Effect of driving experience on anticipatory look-ahead fixations in real curve driving. Accident Analysis \& Prevention

Corresponding author: esko.lehtonen@helsinki.fi

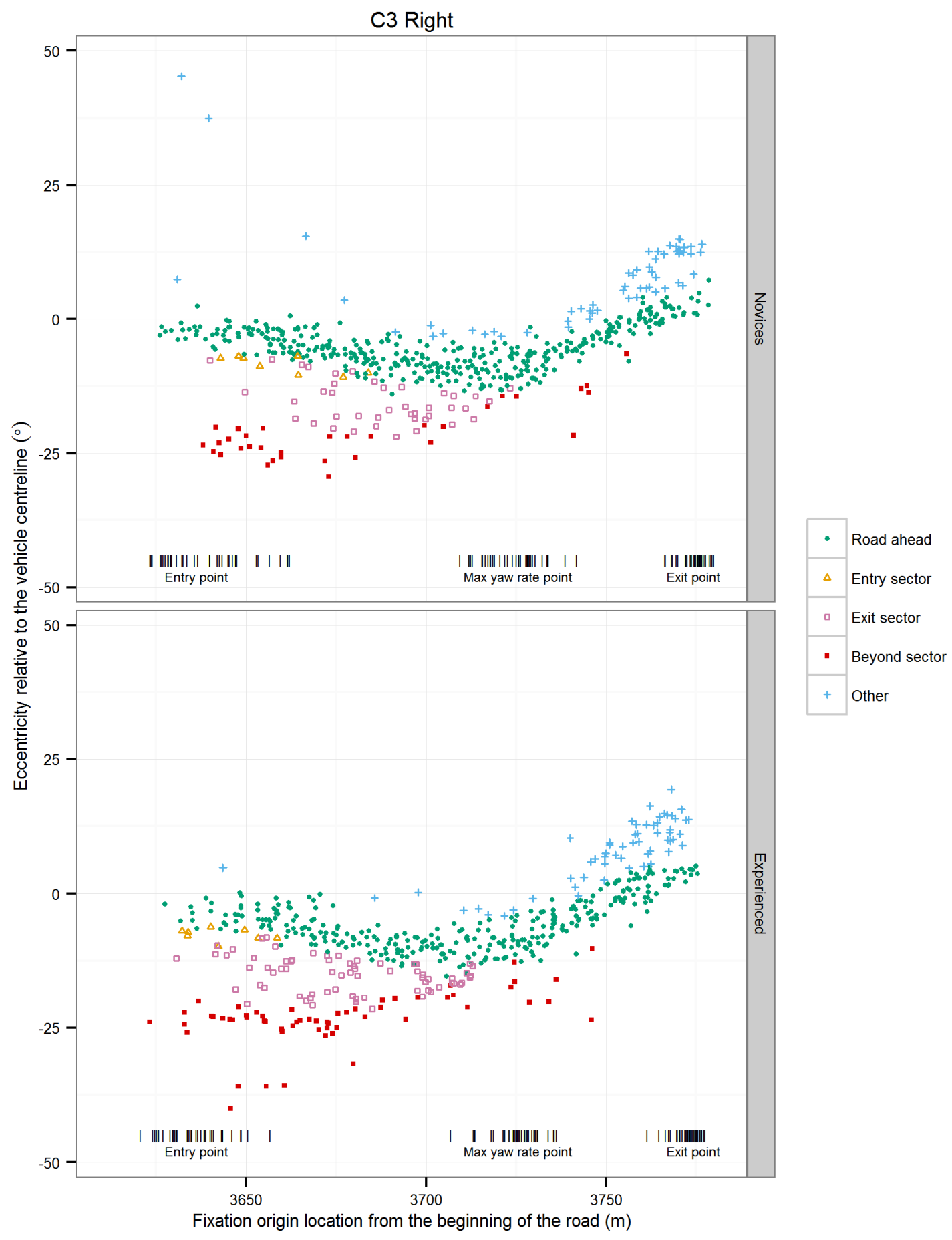

Supplementary Figure 4. Fixations with their origin location ( $x$-scale) and eccentricity relative to the vehicle centreline ( $y$ scale) with a classification (colour and shape), for experience groups (novices, experienced) at curve C3 to right (down). Fixations to road ahead, entry, exit and beyond sectors target the road. Other fixations include those to instrument panels, mirrors, and the scenery. Fixations in different sectors may mix due to differences in individual driving lines, which affect sector boundaries. Distribution of entry, max yaw rate and exit points are marked with vertical lines. 
Supplementary material for Lehtonen, E., Lappi, O., Koirikivi, I., Summala, H. Effect of driving experience on anticipatory look-ahead fixations in real curve driving. Accident Analysis \& Prevention

Corresponding author: esko.lehtonen@helsinki.fi

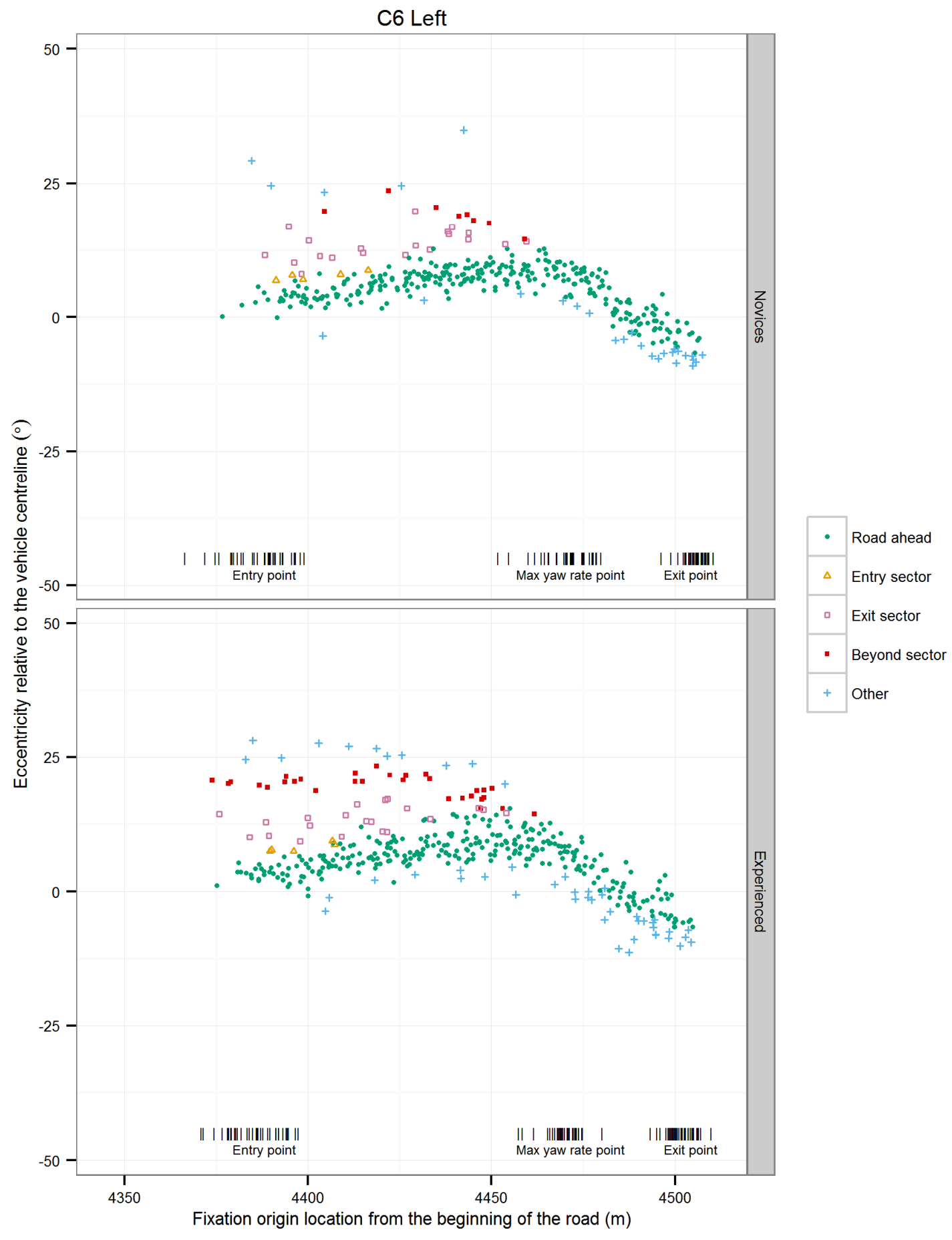

Supplementary Figure 5. Fixations with their origin location ( $x$-scale) and eccentricity relative to the vehicle centreline ( $y$ scale) with a classification (colour and shape), for experience groups (novices, experienced) at curve C6 to left (up). Fixations to road ahead, entry, exit and beyond sectors target the road. Other fixations include those to instrument panels, mirrors, and the scenery. Fixations in different sectors may mix due to differences in individual driving lines, which affect sector boundaries. Distribution of entry, max yaw rate and exit points are marked with vertical lines. 
Supplementary material for Lehtonen, E., Lappi, O., Koirikivi, I., Summala, H. Effect of driving experience on anticipatory look-ahead fixations in real curve driving. Accident Analysis \& Prevention

Corresponding author: esko.lehtonen@helsinki.fi

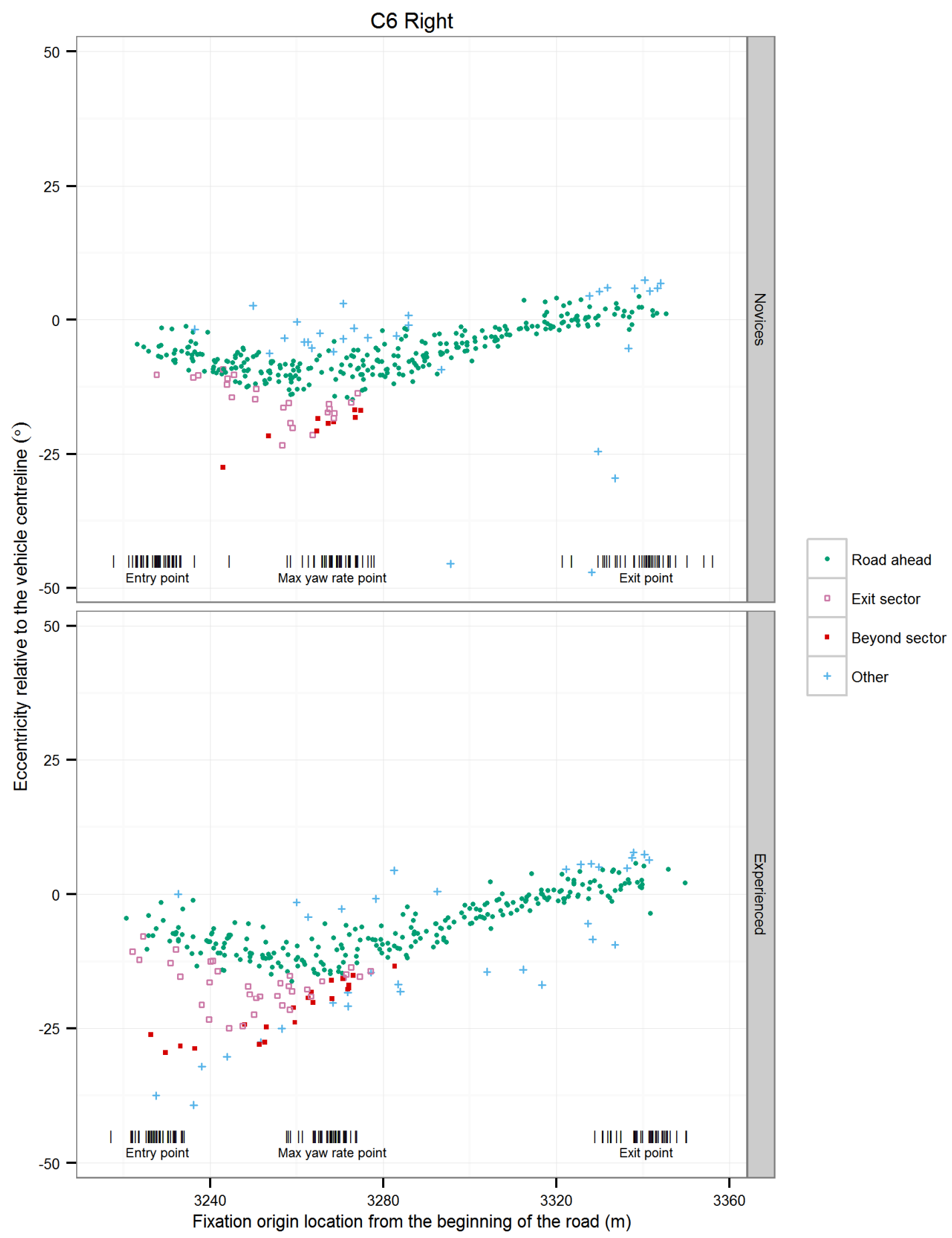

Supplementary Figure 6. Fixations with their origin location ( $\mathrm{x}$-scale) and eccentricity relative to the vehicle centreline ( $y$ scale) with a classification (colour and shape), for experience groups (novices, experienced) at curve C6 to right (down). Fixations to road ahead, entry, exit and beyond sectors target the road. Other fixations include those to instrument panels, mirrors, and the scenery. Fixations in different sectors may mix due to differences in individual driving lines, which affect sector boundaries. Distribution of entry, max yaw rate and exit points are marked with vertical lines. 
Supplementary material for Lehtonen, E., Lappi, O., Koirikivi, I., Summala, H. Effect of driving experience on anticipatory look-ahead fixations in real curve driving. Accident Analysis \& Prevention

Corresponding author: esko.lehtonen@helsinki.fi

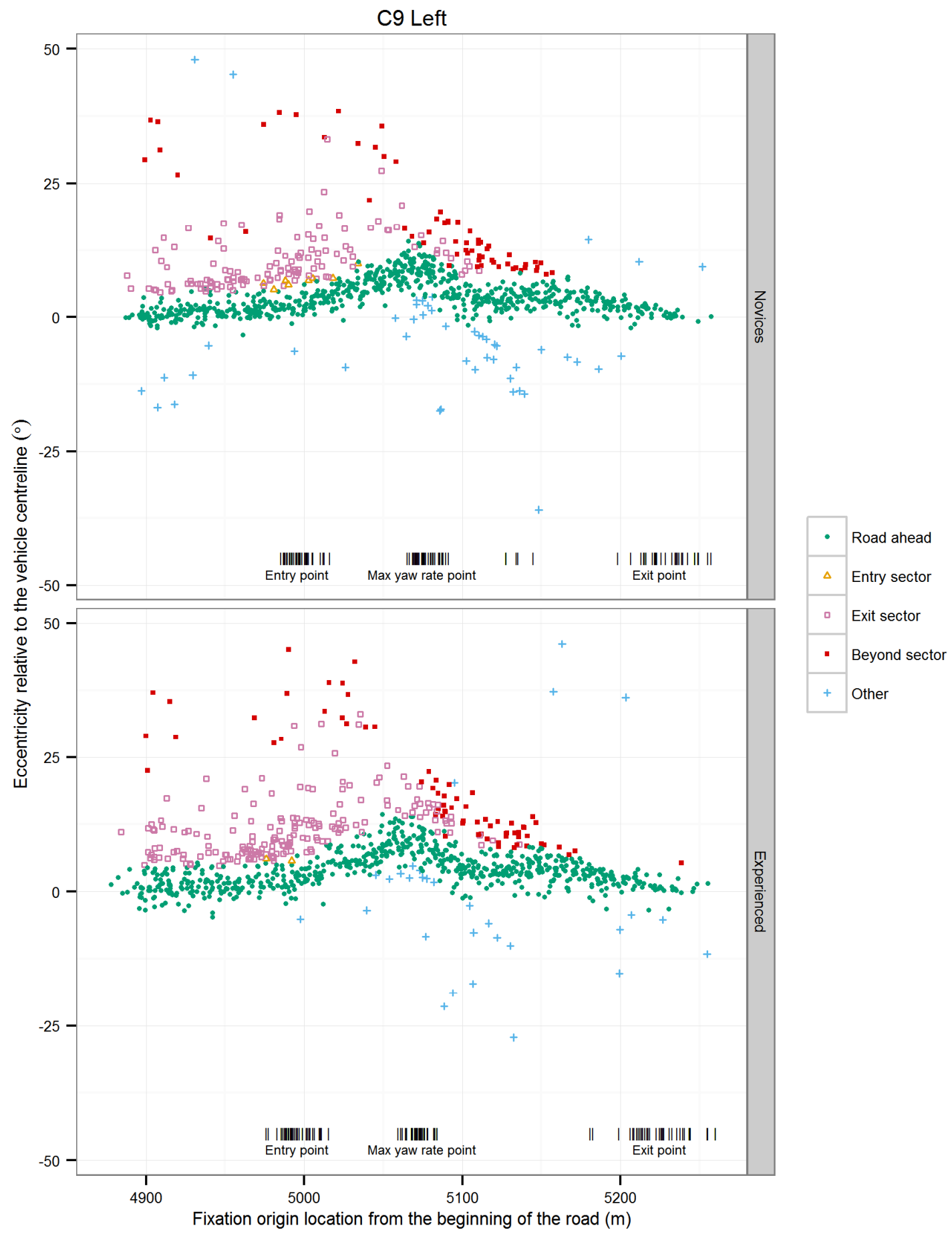

Supplementary Figure 7. Fixations with their origin location ( $x$-scale) and eccentricity relative to the vehicle centreline (yscale) with a classification (colour and shape), for experience groups (novices, experienced) at curve C9 to left (up). Fixations to road ahead, entry, exit and beyond sectors target the road. Other fixations include those to instrument panels, mirrors, and the scenery. Fixations in different sectors may mix due to differences in individual driving lines, which affect sector boundaries. Distribution of entry, max yaw rate and exit points are marked with vertical lines. 
Supplementary material for Lehtonen, E., Lappi, O., Koirikivi, I., Summala, H. Effect of driving experience on anticipatory look-ahead fixations in real curve driving. Accident Analysis \& Prevention

Corresponding author: esko.lehtonen@helsinki.fi

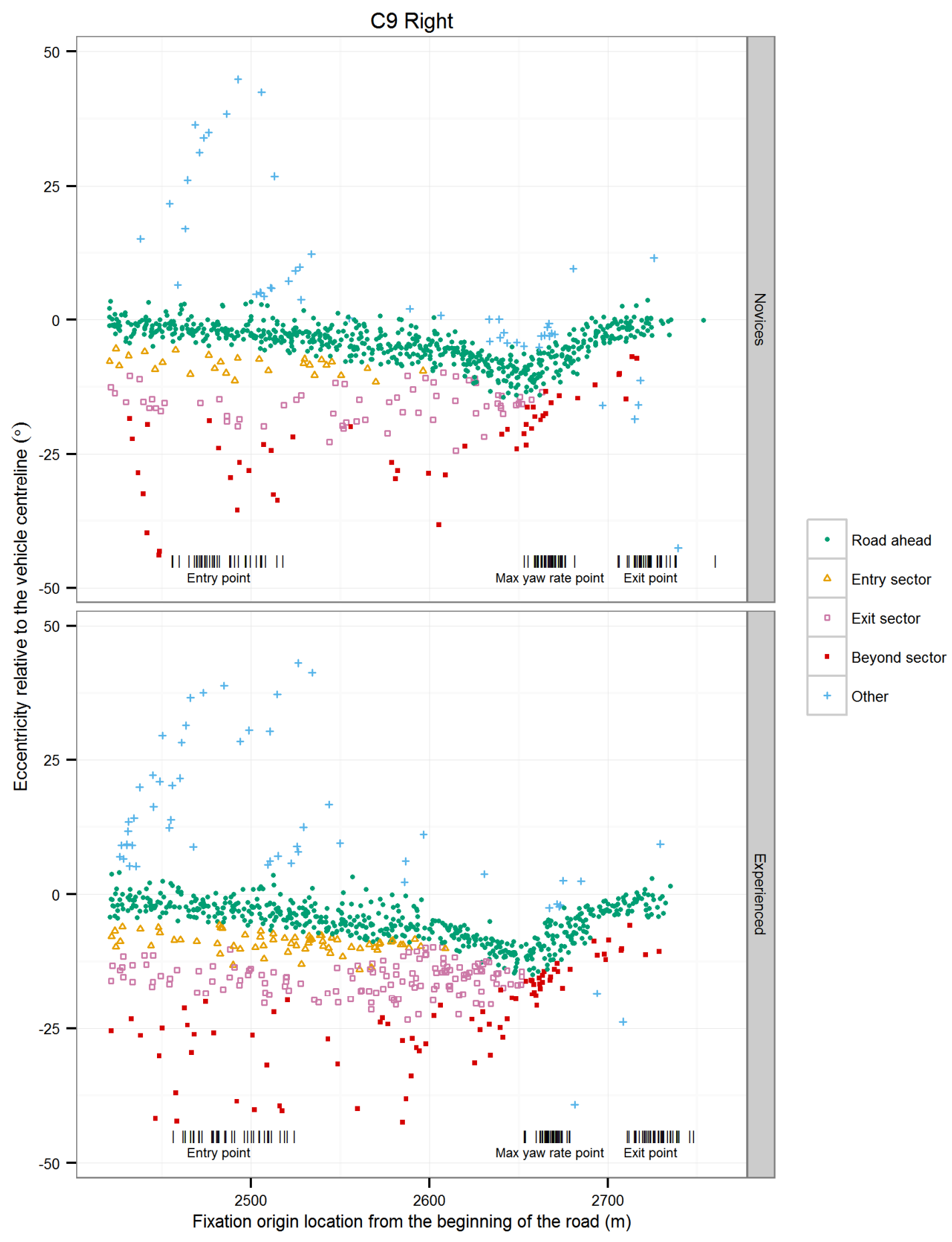

Supplementary Figure 8. Fixations with their origin location ( $\mathrm{x}$-scale) and eccentricity relative to the vehicle centreline ( $y$ scale) with a classification (colour and shape), for experience groups (novices, experienced) at curve C9 to right (down). Fixations to road ahead, entry, exit and beyond sectors target the road. Other fixations include those to instrument panels, mirrors, and the scenery. Fixations in different sectors may mix due to differences in individual driving lines, which affect sector boundaries. Distribution of entry, max yaw rate and exit points are marked with vertical lines. 
Supplementary material for Lehtonen, E., Lappi, O., Koirikivi, I., Summala, H. Effect of driving experience on anticipatory look-ahead fixations in real curve driving. Accident Analysis \& Prevention

Corresponding author: esko.lehtonen@helsinki.fi

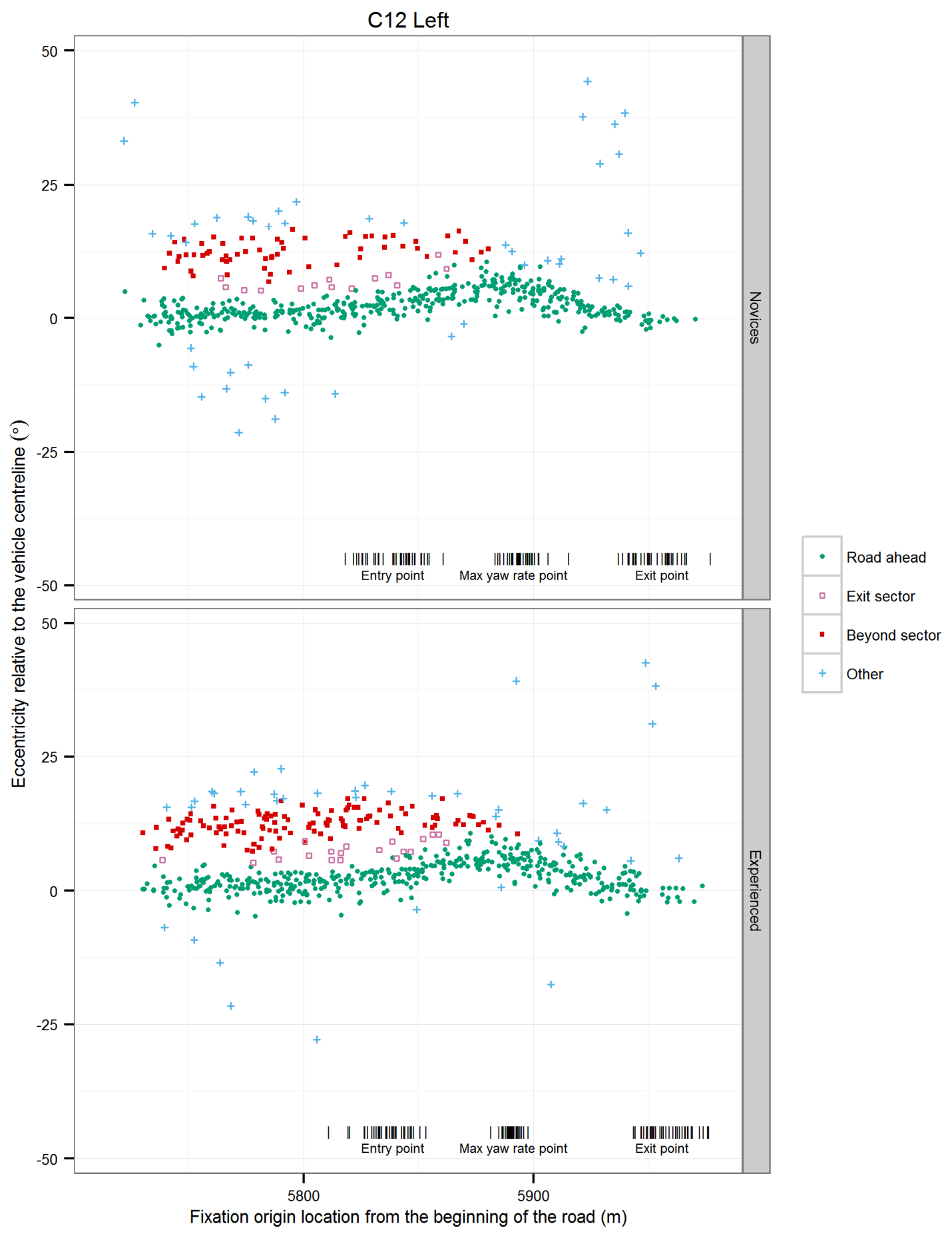

Supplementary Figure 9. Fixations with their origin location ( $x$-scale) and eccentricity relative to the vehicle centreline ( $y$ scale) with a classification (colour and shape), for experience groups (novices, experienced) at curve C12 to left (up). Fixations to road ahead, entry, exit and beyond sectors target the road. Other fixations include those to instrument panels, mirrors, and the scenery. Fixations in different sectors may mix due to differences in individual driving lines, which affect sector boundaries. Distribution of entry, max yaw rate and exit points are marked with vertical lines. 
Supplementary material for Lehtonen, E., Lappi, O., Koirikivi, I., Summala, H. Effect of driving experience on anticipatory look-ahead fixations in real curve driving. Accident Analysis \& Prevention

Corresponding author: esko.lehtonen@helsinki.fi

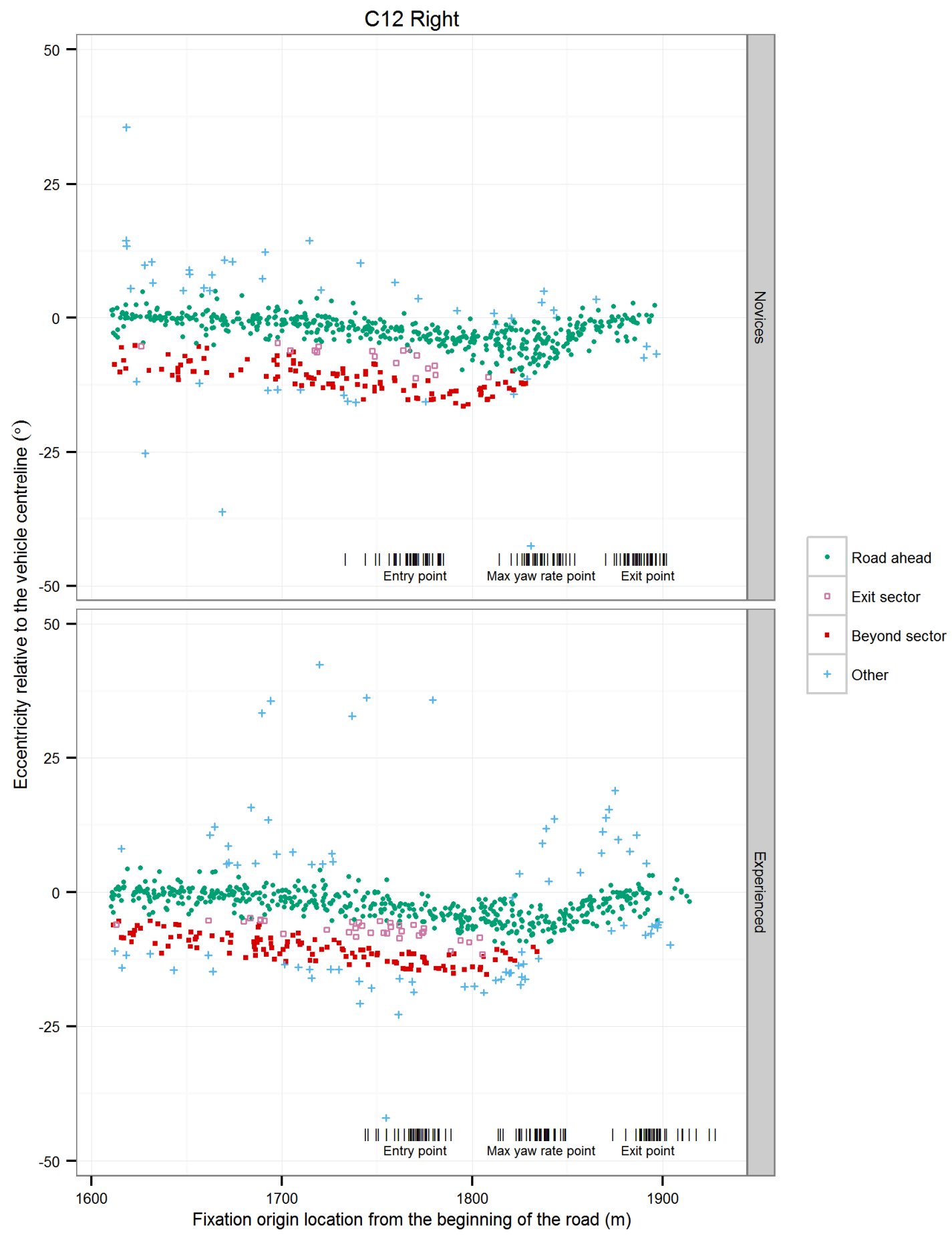

Supplementary Figure 10. Fixations with their origin location ( $x$-scale) and eccentricity relative to the vehicle centreline ( $y$ scale) with a classification (colour and shape), for experience groups (novices, experienced) at curve C12 to right (down). Fixations to road ahead, entry, exit and beyond sectors target the road. Other fixations include those to instrument panels, mirrors, and the scenery. Fixations in different sectors may mix due to differences in individual driving lines, which affect sector boundaries. Distribution of entry, max yaw rate and exit points are marked with vertical lines. 
Supplementary material for Lehtonen, E., Lappi, O., Koirikivi, I., Summala, H. Effect of driving experience on anticipatory look-ahead fixations in real curve driving. Accident Analysis \& Prevention

Corresponding author: esko.lehtonen@helsinki.fi

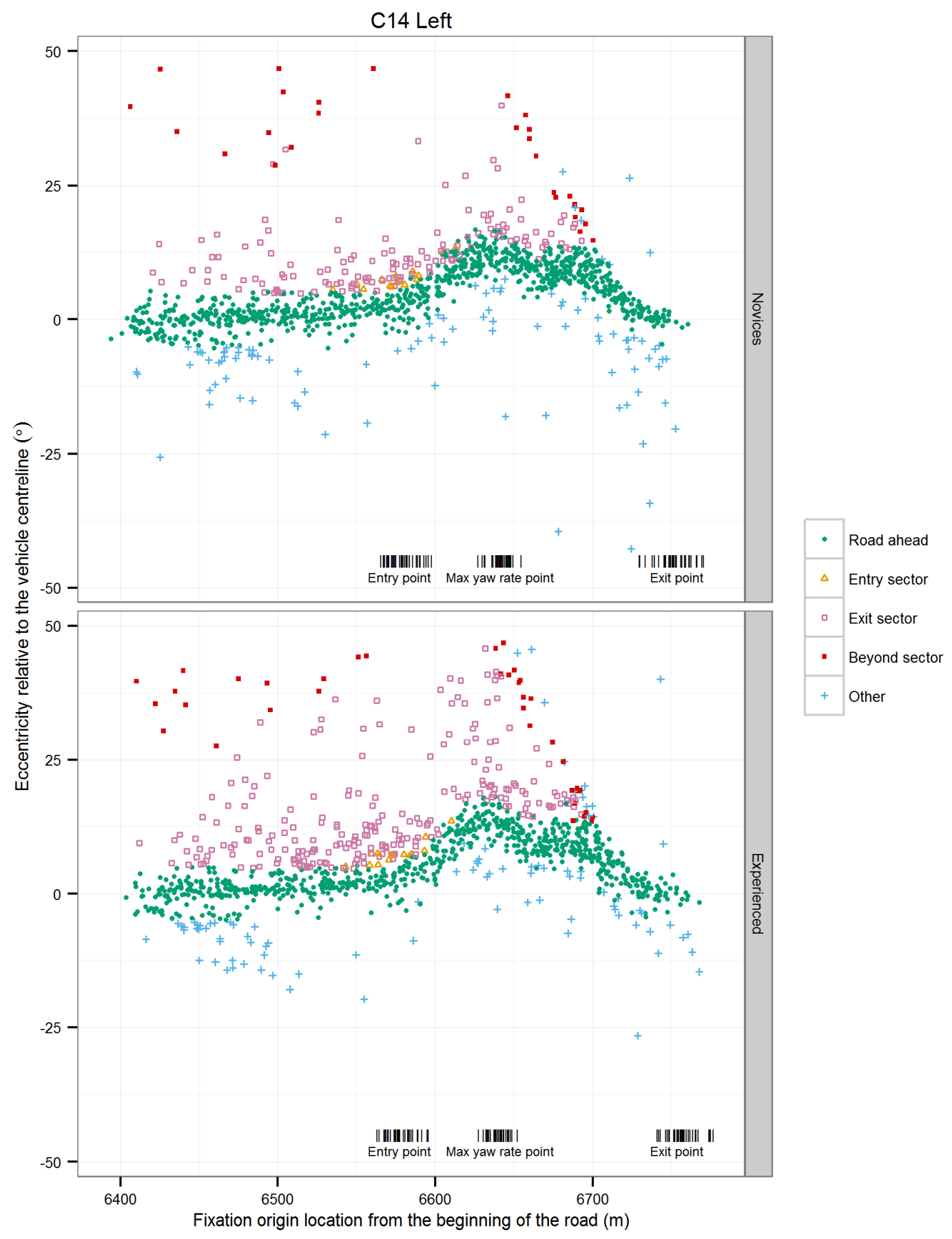

Supplementary Figure 11. Fixations with their origin location ( $x$-scale) and eccentricity relative to the vehicle centreline (yscale) with a classification (colour and shape), for experience groups (novices, experienced) at curve C14 to left (up). Fixations to road ahead, entry, exit and beyond sectors target the road. Other fixations include those to instrument panels, mirrors, and the scenery. Fixations in different sectors may mix due to differences in individual driving lines, which affect sector boundaries. Distribution of entry, max yaw rate and exit points are marked with vertical lines. 
Supplementary material for Lehtonen, E., Lappi, O., Koirikivi, I., Summala, H. Effect of driving experience on anticipatory look-ahead fixations in real curve driving. Accident Analysis \& Prevention

Corresponding author: esko.lehtonen@helsinki.fi

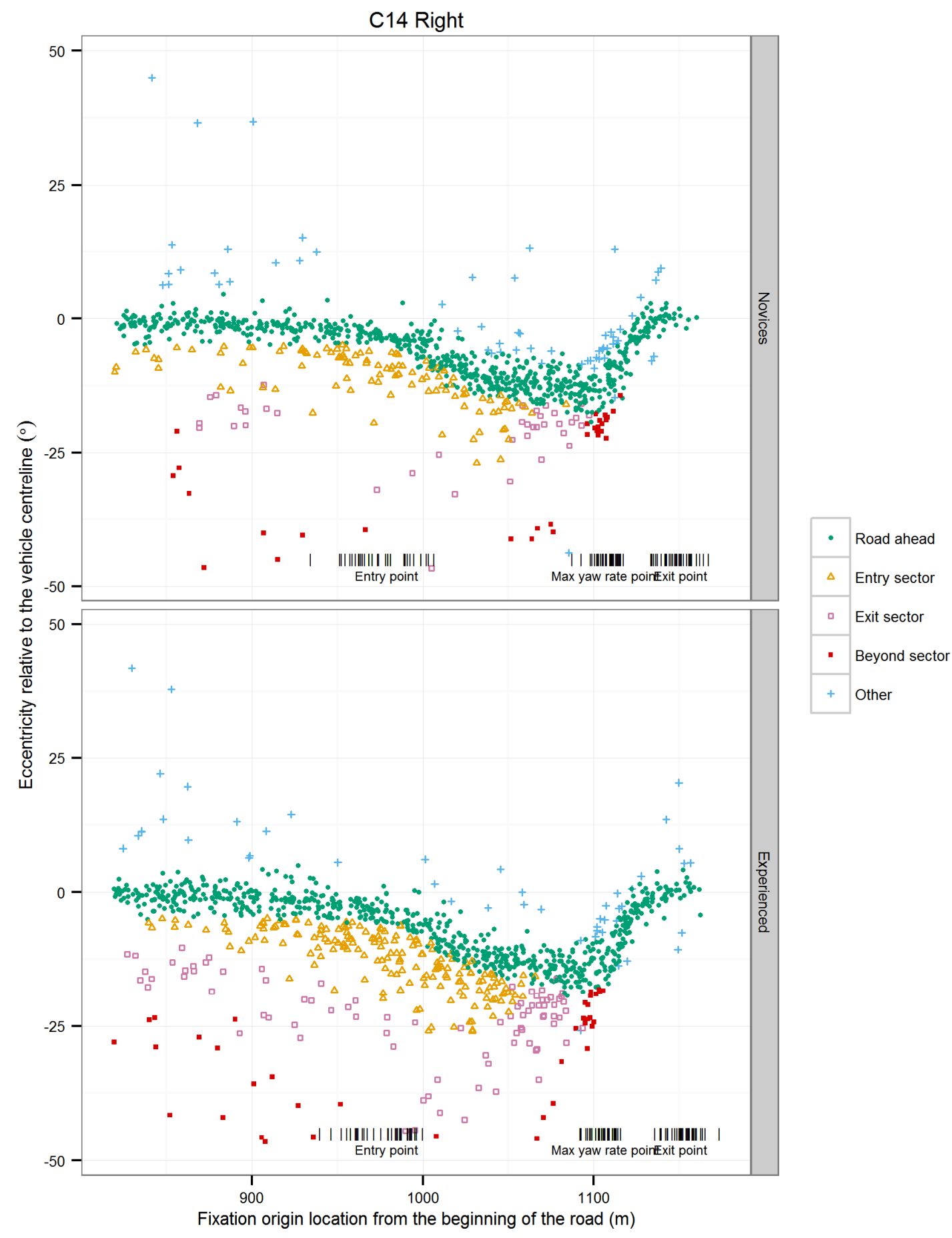

Supplementary Figure 12. Fixations with their origin location ( $x$-scale) and eccentricity relative to the vehicle centreline ( $y$ scale) with a classification (colour and shape), for experience groups (novices, experienced) at curve C14 to right (down). Fixations to road ahead, entry, exit and beyond sectors target the road. Other fixations include those to instrument panels, mirrors, and the scenery. Fixations in different sectors may mix due to differences in individual driving lines, which affect sector boundaries. Distribution of entry, max yaw rate and exit points are marked with vertical lines. 
Supplementary material for Lehtonen, E., Lappi, O., Koirikivi, I., Summala, H. Effect of driving experience on anticipatory look-ahead fixations in real curve driving. Accident Analysis \& Prevention

Corresponding author: esko.lehtonen@helsinki.fi

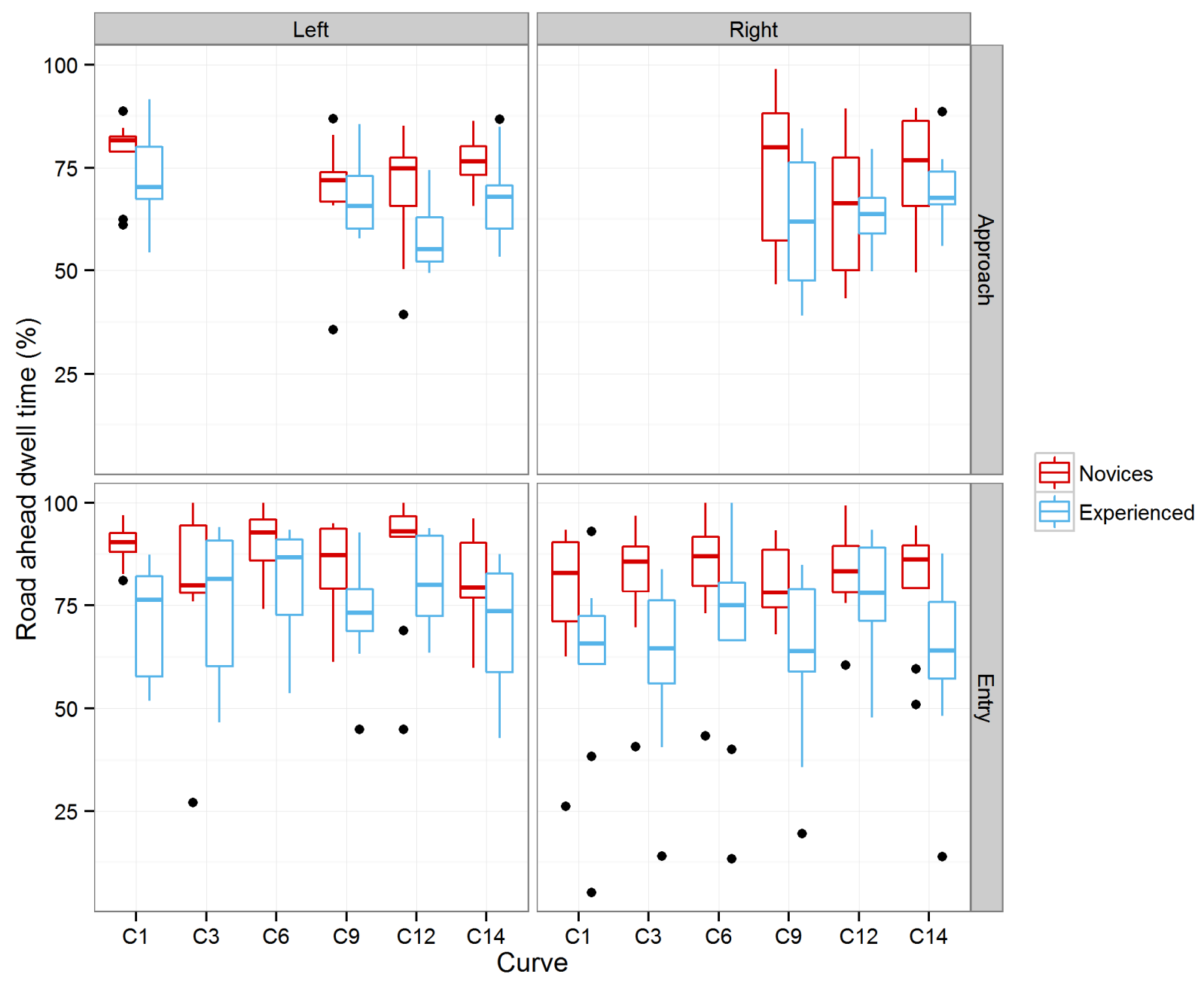

Supplementary Figure 13. Boxplots of road-ahead dwell time percentages on different curves, for curve phases (approach, entry) and driver groups (novices, experienced). Not all curves have an approach phase. 
Supplementary material for Lehtonen, E., Lappi, O., Koirikivi, I., Summala, H. Effect of driving experience on anticipatory look-ahead fixations in real curve driving. Accident Analysis \& Prevention

Corresponding author: esko.lehtonen@helsinki.fi

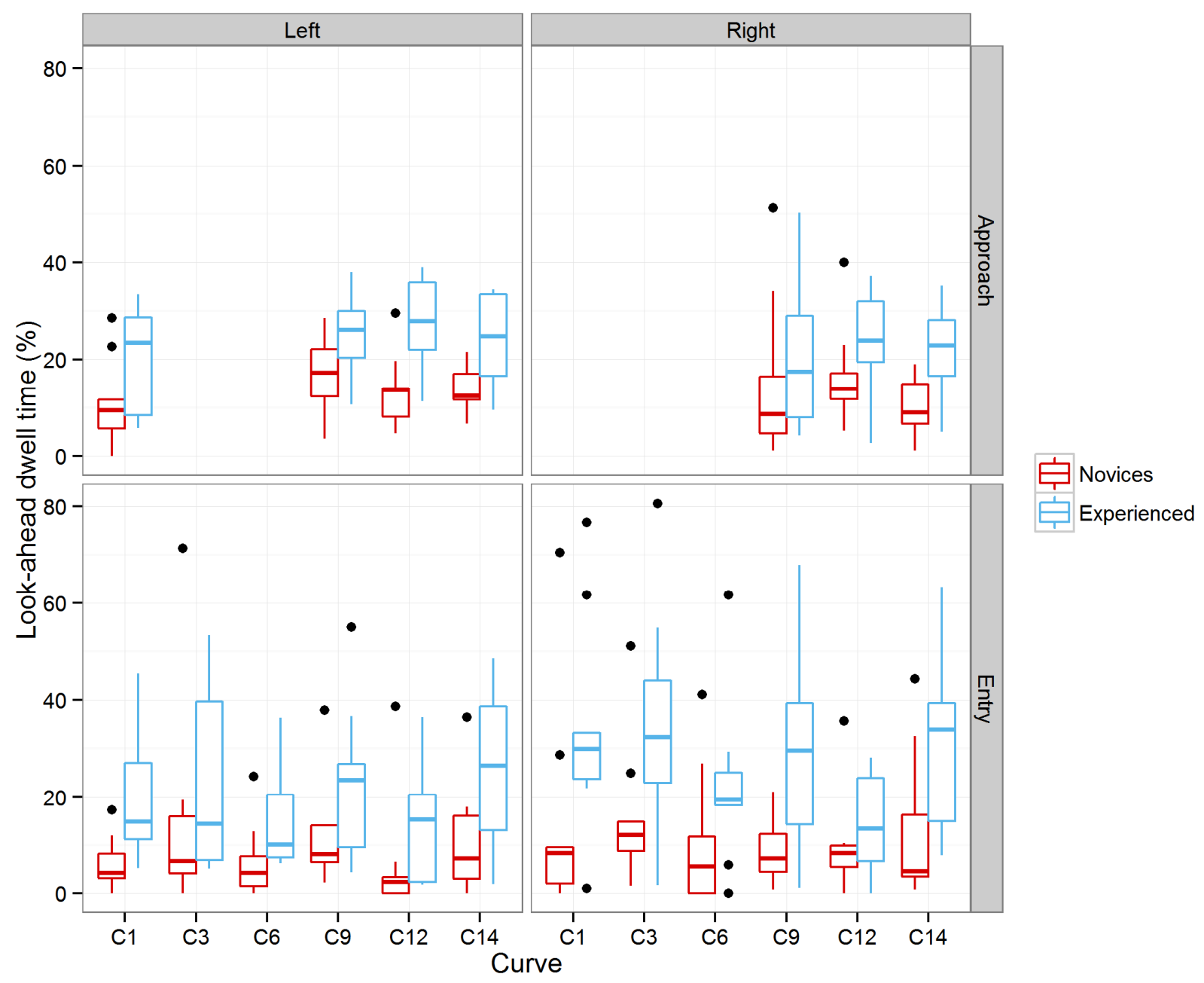

Supplementary Figure 14. Boxplots of look-ahead dwell time on different curves, for curve phases (approach, entry) and driver groups (novices, experienced). Not all curves have an approach phase. 
Supplementary material for Lehtonen, E., Lappi, O., Koirikivi, I., Summala, H. Effect of driving experience on anticipatory look-ahead fixations in real curve driving. Accident Analysis \& Prevention

Corresponding author: esko.lehtonen@helsinki.fi

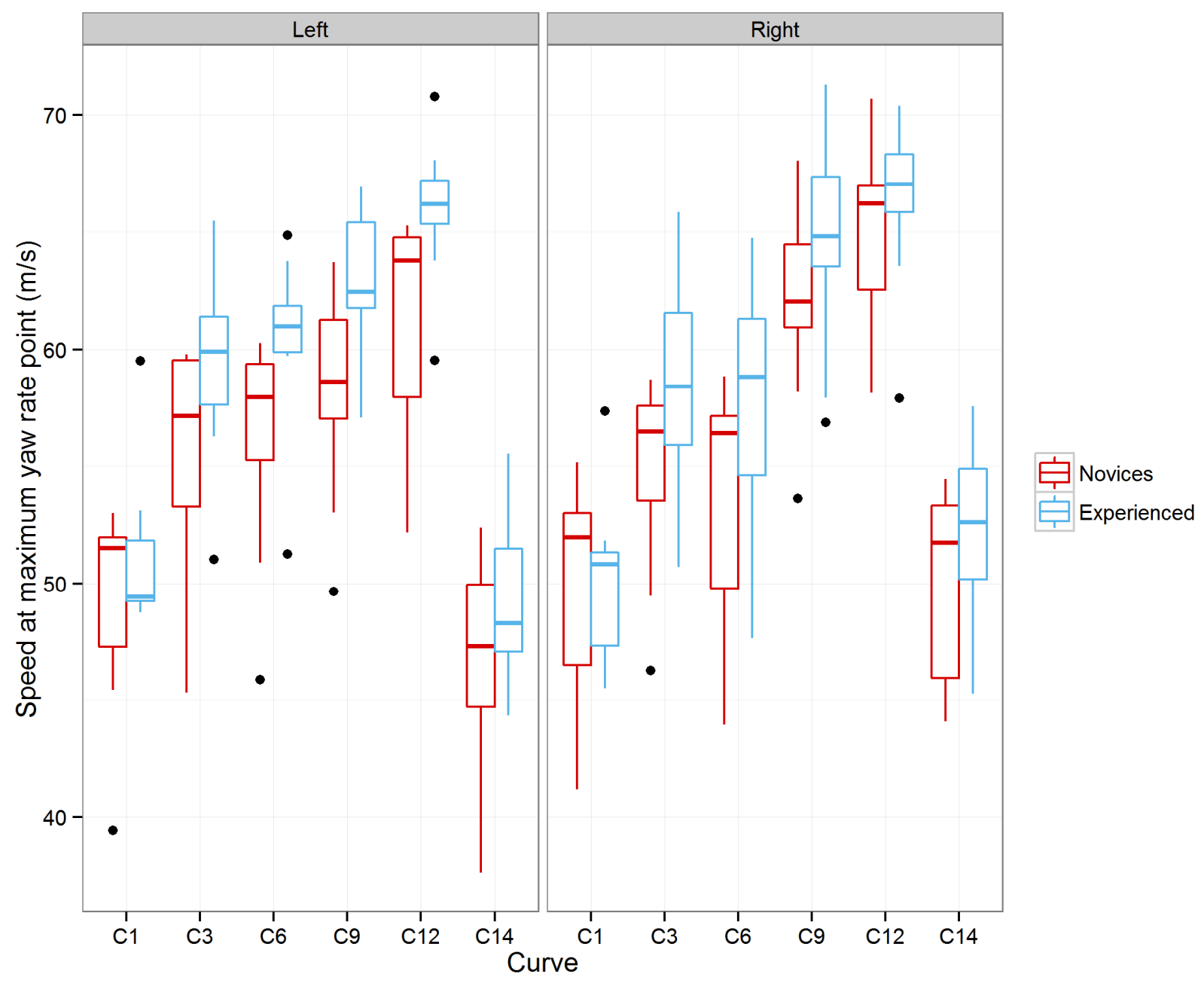

Supplementary Figure 15. Boxplots of vehicle speed at the maximum yaw rate point, for curves and driver groups (novices, experienced). 
Supplementary material for Lehtonen, E., Lappi, O., Koirikivi, I., Summala, H. Effect of driving experience on anticipatory look-ahead fixations in real curve driving. Accident Analysis \& Prevention

Corresponding author: esko.lehtonen@helsinki.fi

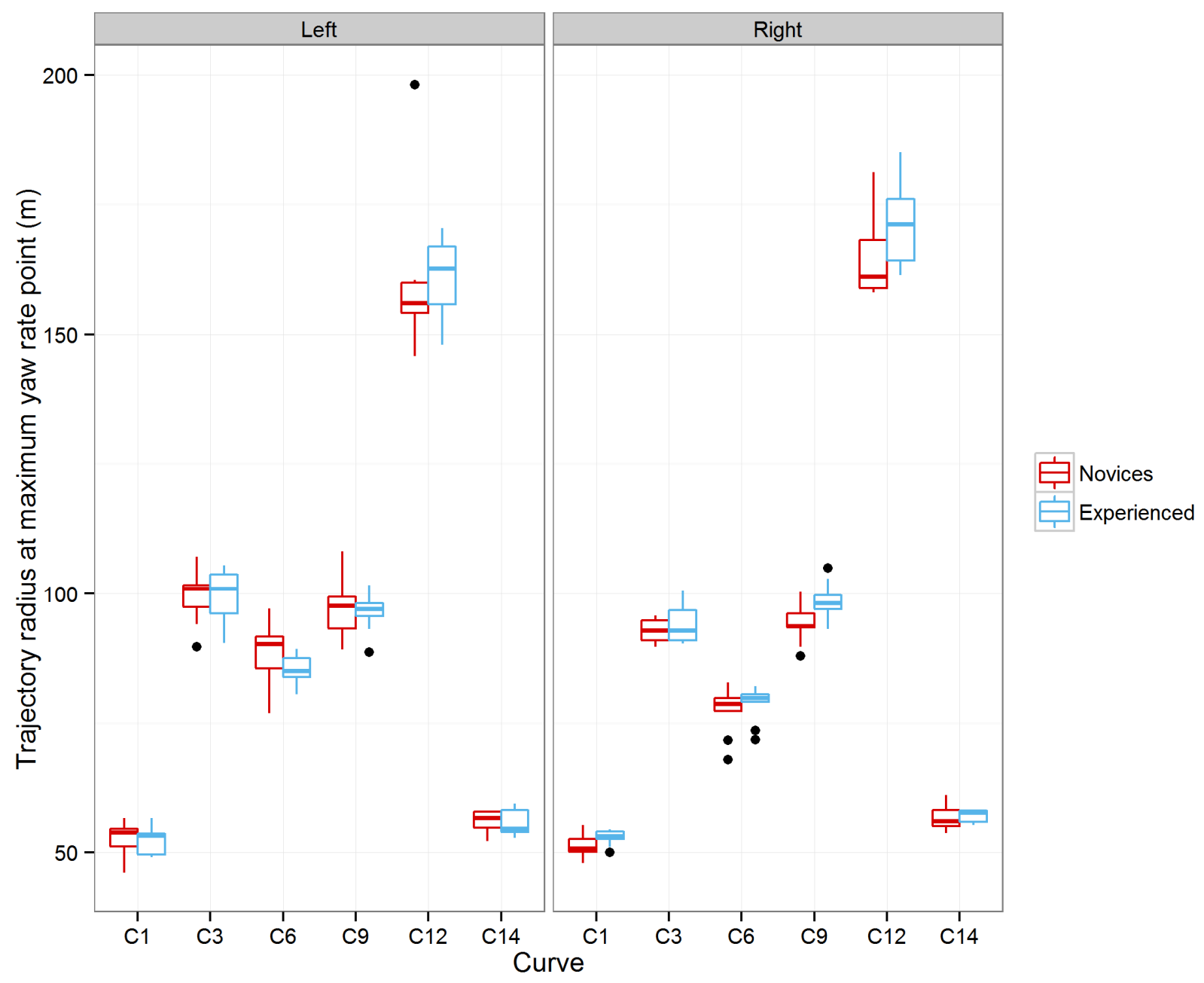

Supplementary Figure 16. Boxplot of the radius of vehicle trajectory at the curve yaw max point, for driver groups (novices, experienced). Radius is calculated by dividing the velocity by the yaw rate at the curve maximum yaw rate point. 